\title{
Origine du bruit dans les dispositifs à semiconducteurs
}

\author{
J. P. Nougier \\ Centre d'Electronique de Montpellier (*), Université des Sciences et Techniques du Languedoc, \\ 34060 Montpellier Cedex, France
}

(Reçu le 16 janvier 1987, accepté le 6 avril 1987)

\begin{abstract}
Résumé. - Après avoir donné les principales définitions et grandeurs physiques liées au bruit, on indique les principales sources de bruit dans les semiconducteurs. On montre ensuite l'intérêt des mesures de bruit dans les matériaux, en tant que moyen de caractérisation technologique et physique. On indique ensuite quelles sont les méthodes modernes de modélisation du bruit des composants, et on termine en mettant l'accent sur les problèmes spécifiques rencontrés dans les composants submicroniques.
\end{abstract}

\begin{abstract}
The main definitions and quantities of physical interest, related to noise measurements, are first given. The noise sources involved in semiconductors are given. The interest of noise measurements in semiconducting materials are pointed out, as a way for getting informations on both technological aspects and transport parameters. The modern ways for modelling the noise of devices are then given, and the specific problems arising in submicron devices are indicated.
\end{abstract}

\section{Introduction.}

Le bruit dans les composants, est dû à des événements aléatoires locaux, produits par les mécanismes de collision des porteurs, dont la trajectoire est aléatoire autour d'une trajectoire moyenne: ceci engendre des fluctuations du courant, et de la tension, autour des valeurs moyennes (point de polarisation). Les mesures de bruit sont importantes, essentiellement pour deux raisons : d'un point de vue fondamental, elles donnent des informations sur les mécanismes de transport (limitations fondamentales telles que les interactions sur les phonons, ou limitations technologiques telles que les interactions sur les défauts et impuretés, etc...) ; d'un point de vue appliqué, le bruit est une limitation fondamentale de la sensibilité ou de la détectivité d'un composant, de sorte qu'il est important de l'étudier pour savoir s'il peut être diminué ou non.

Toute la philosophie qui sous-tend la modélisation du bruit dans les composants, est incluse dans la figure 1 qui montre un composant, polarisé en continu entre les électrodes $\mathbf{M}$ et $\mathbf{N}$, parcouru par un courant continu d'eau (ou d'électrons dans un vrai composant). Le pêcheur, assis en $M^{\prime}$, regarde le

(*) Laboratoire associé au C.N.R.S., U.A. 391. bouchon en $\mathrm{N}^{\prime}$, qui sautille à la surface de l'eau à cause des fluctuations du courant, dues aux cailloux jetés en S. Ces cailloux représentent une source locale de bruit, et le mouvement du bouchon représente le potentiel fluctuant entre les électrodes $\mathrm{M}^{\prime}$ et $\mathrm{N}^{\prime}$, superposé à la polarisation continue. On

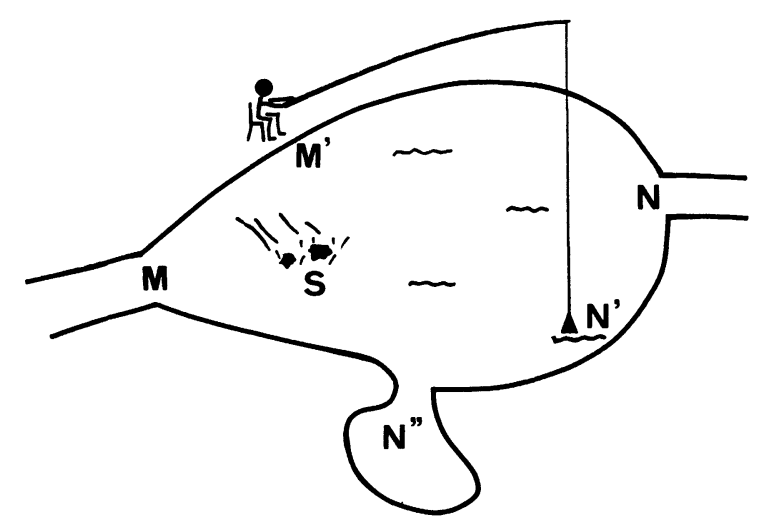

Fig. 1. - Mesure du bruit d'un composant. $M$ et $N$, électrodes de polarisation. $\mathbf{M}^{\prime}, \mathrm{N}^{\prime}, \mathrm{N}^{\prime \prime}$, électrodes de mesure. S, source de bruit.

[Electronic-like device for noise measurement. $\mathrm{M}$ and $\mathrm{N}$ are the d.c. electrodes. $\mathrm{M}^{\prime}, \mathrm{N}^{\prime}$, and $\mathrm{N}^{\prime \prime}$ are the a.c. electrodes. $\mathrm{S}$ is a noise source.] 
voit de suite que les électrodes $\mathrm{M}^{\prime}$ et $\mathrm{N}^{\prime}$ où on étudie le bruit peuvent être ou non confondues avec les électrodes $\mathrm{M}$ et $\mathrm{N}$ de polarisation du composant. $\mathbf{M}^{\prime}$ sera pris comme potentiel de référence pour les fluctuations (masse en courant alternatif).

Il est évident, d'après la figure 1, que le bruit mesuré entre $\mathbf{M}^{\prime}$ et $\mathbf{N}^{\prime}$, dépend d'une part de la source locale du bruit en $\mathrm{S}$, d'autre part de la manière dont les fluctuations, créées en $\mathrm{S}$ par la source de bruit, se propagent en $\mathrm{N}^{\prime}$ (si le bouchon était placé en $\mathrm{N}^{\prime \prime}$, il tremblerait moins) : la fonction de transfert entre la source locale et les électrodes de mesure est appelée champ d'impédances. Le bruit total, mesuré entre les électrodes $\mathrm{M}^{\prime}$ et $\mathrm{N}^{\prime}$, est la somme des fluctuations produites par toutes les sources de bruit localisées aux différents points du composant.

Dans cet article, je donnerai les définitions fondamentales des quantités relatives au bruit ( $\$ 2)$, puis les expressions des différentes sources de bruit ( $\$ 3$ ). Je montrerai, sur un petit nombre d'exemples, l'intérêt des mesures de bruit dans les matériaux (§ 4). La méthode du champ d'impédance sera exposée au $\S 5$, et je terminerai en indiquant quels sont les problèmes spécifiques soulevés par les composants submicroniques $(\S 6)$.

\section{Définitions et grandeurs liées au bruit.}

2.1 FONCTIONS ALÉATOIRES ET DENSITÉS SPECTRALES. - Une fonction certaine du temps $F_{\mathrm{c}}(t)$ peut se développer en intégrale de Fourier. Soit $\bar{F}_{\mathrm{c}}(t)^{t}$ sa valeur moyenne. La composante de $F_{\mathrm{c}}(t)-{\overline{F_{\mathrm{c}}(t)}}^{t}$ à la fréquence $f$, dans la bande $\Delta f$, possède une amplitude $a(f)$ constante, indépendante du temps.

Considérons maintenant une fonction aléatoire $F_{\mathrm{a}}(t)$, de valeur moyenne $F_{0}={\overline{F_{\mathrm{a}}(t)}}^{t}$. Si on développe $F_{\mathrm{a}}(t)-F_{0}$ en intégrale de Fourier (ou plus rigoureusement de Fourier-Stieltjes), la composante à la fréquence $f$, dans la bande $\Delta f$, a une amplitude $a(f, t)$ qui change de façon soudaine en même temps que $F_{\mathrm{a}}(t)$, c'est-à-dire à des instants aléatoires

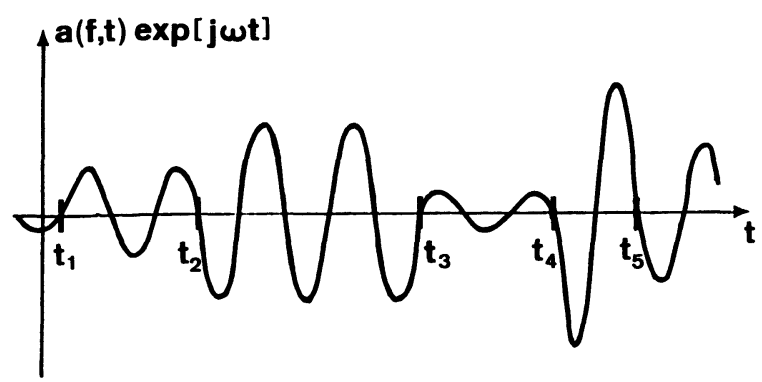

Fig. 2. - Variation, en fonction du temps, de la composante $a(f, t) \exp j \omega t$ de la fonction aléatoire $F_{\mathrm{a}}(t)$.

[Variation, versus time, of the Fourier component $a(f, t) \exp j \omega t$, of the random function $F_{\mathrm{a}}(t)$.] $t_{1}, t_{2}, \ldots, t_{j}, \ldots$ (voir Fig. 2). Deux quantités entre autres sont intéressantes, en particulier pour les fonctions aléatoires stationnaires d'ordre 2 :

a) L'amplitude moyenne, ou plutôt sa valeur quadratique moyenne

$$
S_{F}(f) \Delta f=\overline{|a(f, t)|^{2}}=\overline{a(f, t) \cdot a^{*}(f, t)}{ }^{t}
$$

où * signifie la conjugaison complexe, et où la moyenne est prise sur le temps, ou sur un ensemble statistique lorsqu'il y a ergodicité. $S_{F}(f)$ est la densité spectrale de fluctuations de $F_{\mathrm{a}}(t)$.

b) Le temps moyen $\tau_{\mathrm{c}}=\overline{t_{j+1}-t_{j}}$. Pour des durées $t \ll \tau_{\mathrm{c}}, f_{\mathrm{a}}(t)$ et donc $a(f, t)$ reste constant. Pour des durées $t \gg \tau_{c}$, l'amplitude change de façon imprévisible. Ainsi, $\tau_{\mathrm{c}}$ est le temps de corrélation de la fonction aléatoire $F_{\mathrm{a}}(t)$.

On obtient ainsi une première méthode de mesure de bruit de la quantité à étudier $F_{\mathrm{a}}(t)$ (qui sera en pratique un courant ou une tension) : par filtrage à la fréquence $f$ dans la bande $\Delta f$, on obtient $a(f, t)$. Puis une détection quadratique donne $\left|a^{2}(f, t)\right|$, et une intégration donne un signal de sortie proportionnel à ${\overline{\left|a^{2}(f, t)\right|}}^{t}$, c'est-à-dire proportionnel à $S_{F}(f)$.

Nous avons donné la définition physique de $S_{F}(f)$. En fait, on définit $S_{F}(f)$ comme transformée de Fourier de la fonction de corrélation de $F_{\mathrm{a}}(t)$. Soit $\Delta F(t)$ l'écart instantané de $F_{\mathrm{a}}(t)$ par rapport à sa valeur moyenne $F_{0}={\overline{F_{\mathrm{a}}(t)}}^{t}$ :

$$
F_{\mathrm{a}}(t)=F_{0}+\Delta F(t) .
$$

La fonction de corrélation de $F_{\mathrm{a}}(t)$ est définie par :

$$
\Gamma_{F}\left(F_{0}, \theta\right)=\overline{\Delta F(t) \cdot \Delta F^{*}(t-\theta)} .
$$

La densité spectrale à la fréquence mathématique (c'est-à-dire algébrique) $\nu$, vaut :

$$
\gamma_{F}\left(F_{0}, \nu\right)=\int_{-\infty}^{+\infty} \Gamma_{F}\left(F_{0}, \theta\right) \exp (-2 j \pi \nu \theta) \mathrm{d} \theta
$$

et la densité spectrale mesurable, à la fréquence physique positive $f$, vaut :

$$
S_{F}\left(F_{0}, f\right)=\gamma_{F}\left(F_{0}, \nu=f\right)+\gamma_{F}\left(F_{0}, \nu=-f\right) \text {. }
$$

Dans le cas usuel des fonctions aléatoires $F_{\mathrm{a}}(t)$ réelles, l'expression ci-dessus devient :

$$
S_{F}\left(F_{0}, f\right)=4 \int_{0}^{\infty} \Gamma_{F}\left(F_{0}, \theta\right) \cos (2 \pi f \theta) \mathrm{d} \theta
$$

Dans le cas de régimes linéaires, $S_{F}\left(F_{0}, f\right)$ ne dépend pas du point de polarisation $F_{0}$. En régime non linéaire, $S_{F}$ dépend en général de $F_{0}$.

Ceci donne une seconde méthode de mesure de bruit : le signal aléatoire $F_{\mathrm{a}}(t)$ est d'abord échantil- 
lonné, donnant $F_{j}=F_{\mathrm{a}}\left(t_{j}\right)$, puis la fonction de corrélation est calculée, donnant

$$
\Gamma_{k}=\Gamma_{F}\left(\theta_{k}\right)=\sum_{j} F\left(t_{j}\right) \cdot F\left(t_{j}-k \Delta t\right)
$$

et finalement un échantillonnage en fréquence $S_{F}\left(F_{0}, f_{k}\right)$ de la densité spectrale est obtenue par transformée de Fourier discrète de $\Gamma_{k}$.

2.2 QuANTITÉS MESURÉES. - En prenant comme fonction aléatoire $F_{\mathrm{a}}(t)$, soit le courant $I(t)$, soit la tension $V(t)$, on obtient les densités spectrales, plus brièvement appelées courant de bruit $S_{\mathrm{I}}(f)$ et tension de bruit $S_{\mathrm{V}}(f)$, autour du point de polarisation $\left(I_{0}, V_{0}\right)$.

Si on superpose des petits signaux $\delta I \exp j \omega t$ et $\delta V \exp j \omega t$, au point de polarisation $\left(I_{0}, V_{0}\right)$, l'impédance $Z(f)=Z\left(V_{0}, f\right)$ et l'admittance $Y(f)=Y\left(V_{0}, f\right)$ différentielles sont définies par:

$$
Z(f)=\delta V / \delta I \text { et } Y(f)=1 / Z(f) .
$$

Alors, $S_{\mathrm{V}}(f)$ et $S_{\mathrm{I}}(f)$ sont reliés par :

$$
S_{\mathrm{V}}(f)=|Z(f)|^{2} S_{\mathrm{I}}(f) .
$$

La température de bruit $T_{n}(f)=T_{n}\left(V_{0}, f\right)$, autour du point de polarisation $\left(I_{0}, V_{0}\right)$, est définie par l'une des deux relations équivalentes :

$$
\begin{aligned}
& S_{\mathrm{V}}(f)=4 k_{\mathrm{B}} T_{n}(f) \operatorname{Re}\{Z(f)\} \\
& S_{\mathrm{I}}(f)=4 k_{\mathrm{B}} T_{n}(f) \operatorname{Re}\{Y(f)\}
\end{aligned}
$$

où $k_{\mathrm{B}}$ est la constante de Boltzmann, et Re désigne la partie réelle. On peut montrer facilement que $k_{\mathrm{B}} T_{n}$ est la puissance de bruit maximale, par unité de bande passante, qui peut être recueillie dans un circuit extérieur, lorsqu'il y a adaptation d'impédances. Par conséquent, la température de bruit $T_{n}(f)$ peut être mesurée. En fait, en haute fréquence, on mesure effectivement $T_{n}(f)$, et la mesure de $Z(f)$ ou $Y(f)$ permet d'en déduire $S_{\mathrm{V}}(f)$ ou $S_{\mathrm{I}}(f)$ à partir des relations (6) et (7).

\section{Sources de bruit.}

Il ressort de la relation $j=q n v$, que le courant dans un matériau ou un composant fluctue, si l'une au moins des quantités $q, n$, ou $v$, fluctue, produisant ainsi respectivément du bruit de grenaille, du bruit de génération-recombinaison, du bruit de diffusion.

3.1 BRuIT DE GRENAIlle. - Il est dû à l'émission d'électrons à partir de la cathode, à des instants aléatoires Poissoniens. Il en résulte que le flux des charges $q$ n'est pas constant, mais fluctue. Ceci donne lieu à un courant de bruit, observable dans les tubes à vide, les diodes tunnel, etc., et donné par [1] :

$$
S_{\mathrm{I}}(f)=2 q I
$$

où $I$ est le courant moyen. Ce bruit est blanc, aux fréquences habituelles.

3.2 SourCes locales DE BRUIT. - Soit $\mathbf{j}(\mathbf{r}, t)$ la densité de courant locale, de valeur moyenne $\mathbf{j}_{0}(\mathbf{r})$. L'écart $\Delta \mathbf{j}(\mathbf{r}, t)=\mathbf{j}(\mathbf{r}, t)-\mathbf{j}_{0}(\mathbf{r})$ fluctue. Soit $\Delta j_{\alpha}(\mathbf{r}, f, t)$ la composante de Fourier, à la fréquence $f$, dans la bande $\Delta f$, de la projection de $\Delta \mathbf{j}(\mathbf{r}, t)$ sur la direction $\alpha$. Le terme de source de bruit est défini par [2] :

$$
S_{j \alpha \beta}\left(\mathbf{r}, \mathbf{r}^{\prime}, f\right) \Delta f=\overline{\Delta j_{\alpha}(\mathbf{r}, f, t) \cdot \Delta j_{\beta}^{*}\left(\mathbf{r}^{\prime}, f, t\right)^{t}} .
$$

On suppose généralement que les sources de bruit en deux points différents sont décorrélées, ce qui donne :

$$
S_{j \alpha \beta}\left(\mathbf{r}, \mathbf{r}^{\prime}, f\right)=K_{\alpha \beta}(\mathbf{r}, f) \delta\left(\mathbf{r}-\mathbf{r}^{\prime}\right)
$$

où $\delta\left(\mathbf{r}-\mathbf{r}^{\prime}\right)$ désigne la fonction de Dirac. Le terme $K_{\alpha \beta}(\mathbf{r}, f)$ est appelé source locale de bruit. En intégrant les équations (9) et (10) dans la tranche d'épaisseur $\Delta x^{\prime}$ d'un tube de courant alternatif (voir Fig. 3), on obtient la fluctuation du courant à travers les tranches:

$$
\begin{array}{r}
\overline{\Delta I(x, f) \cdot \Delta I^{*}\left(x^{\prime}, f\right)^{t}}= \\
=\Delta A(x) K_{x}(\mathbf{r}, f) \delta\left(x-x^{\prime}\right) \Delta f .
\end{array}
$$

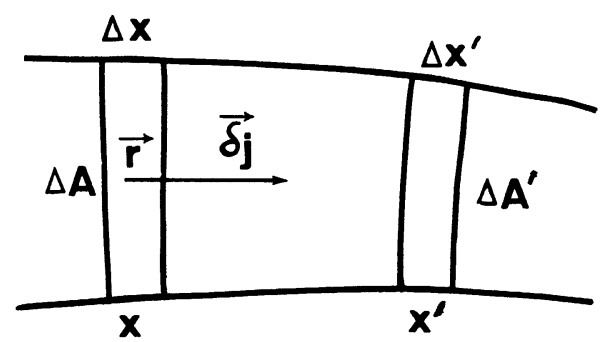

Fig. 3. - Schéma d'un tube de courant ; $x$ est l'abscisse curviligne, le long d'une ligne de courant alternatif, de la tranche de largeur $\Delta x$.

[Slice of a current tube ; $x$ is the abscissa of the slice of thickness $\Delta x$ along an a.c. current path.]

Cette relation, donnée dans la référence [3], avait déjà été établie pour des types particuliers de sources de bruit $[4,5]$. En intégrant l'équation (11) dans la tranche $\Delta x^{\prime}=\Delta x$, on obtient la relation liant la source de bruit locale $K_{x}(\mathbf{r}, f)$ dans la direction $x$, et le courant de bruit $S_{\mathrm{Ix}}(\mathbf{r}, f)$, dans la direction $x$, à travers la tranche située en $\mathbf{r}$ :

$$
K_{x}(\mathbf{r}, f)=S_{\mathrm{Ix}}(\mathbf{r}, f) \Delta x / \Delta A(x) .
$$

La méthode expérimentale de détermination de la source locale de bruit découle de la relation (12) : on mesure le courant de bruit d'un barreau homogène dans un champ électrique uniforme $E$, ce qui donne 
$K_{x}(E, f)$; puis, dans un composant non homogène de même concentration d'impuretés, même orientation cristalline, etc., on pose $K_{x}(\mathbf{r}, f)=K_{x}$ $(E(\mathbf{r}), f)$.

A champ élevé, il faut faire les mesures en impulsions courtes, pour ne pas échauffer le barreau, et donc analyser le bruit en haute fréquence. Dans ces conditions, le paramètre auquel on a accès est la puissance de bruit, c'est-à-dire la température de bruit $T_{n}[6,7]$. Puisque $T_{n}$ est reliée à $S_{\mathrm{I}}$ (cf. Eq. (7)), $K$ peut être reliée à $T_{n}[8]$. Soit $\Delta Y_{x}$ l'impédance différentielle, à la fréquence $f$, de la tranche $\Delta x$. On obtient $[6,7]$, à partir de l'équation (7) :

$$
\begin{gathered}
S_{\mathrm{I}}(r, f)=4 k_{\mathrm{B}} T_{n x}(r, f) \operatorname{Re}\left\{\Delta Y_{x}\right\} \\
\Delta Y_{x}=\delta I / \delta V=\Delta A \delta j_{x}(\mathbf{r}) /\left(\Delta x \delta E_{x}(\mathbf{r})\right) .
\end{gathered}
$$

Les équations (13) et (14), portées dans (12), donnent :

$$
K_{\alpha}(\mathbf{r}, f)=4 k_{\mathrm{B}} T_{n \alpha}(\mathbf{r}, f) \operatorname{Re}\left\{\sigma_{\alpha \alpha}(\mathbf{r}, f)\right\}
$$

où $\overline{\bar{\sigma}}(\mathbf{r}, f)$ est le tenseur de conductivité différentielle à la fréquence $f: \delta \mathbf{j}=\overline{\mathbf{\sigma}} \delta \mathbf{E}$. L'équation (15) montre que la source de bruit peut être déterminée expérimentalement, en mesurant la température de bruit, et la conductivité différentielle, sans aucune hypothèse sur la nature physique de la source de bruit [8]. Notons que la direction $\alpha$ peut être différente de celle le long de laquelle est appliqué le champ de polarisation continue [8].

3.3 BRUIT DE DIFFUSION. - Le bruit de diffusion est dû aux fluctuations des vitesses des porteurs. Soit $\Delta N(\mathbf{r})$ le nombre de porteurs situés dans la tranche de volume $\Delta A \cdot \Delta x$. Le courant à travers cette tranche vaut :

$$
\Delta I=q n(\mathbf{r}) v(\mathbf{r}) \Delta A
$$

où $v(\mathbf{r})$ est la vitesse de dérive, c'est-à-dire la vitesse moyenne des porteurs se trouvant dans la tranche, qui fluctue puisque $\Delta N(\mathbf{r})$ est fini. D'après l'équation (16), on a :

$$
S_{\mathrm{I}}(x, f)=q^{2} n^{2}(\mathbf{r}) \Delta A^{2} S_{v}(\mathbf{r}, f) .
$$

Soit $S_{\mathrm{vi}}(\mathbf{r}, f)$ la densité spectrale de fluctuations de vitesse d'un seul porteur :

$$
\begin{aligned}
S_{\mathrm{v}}(\mathbf{r}, f)=S_{\mathrm{vi}}(\mathbf{r}, f) / \Delta N(\mathbf{r})= \\
=S_{\mathrm{vi}}(\mathbf{r}, f) /(n(r) \Delta A \Delta x) .
\end{aligned}
$$

Puisque, par définition [6,7], $S_{\mathrm{vi}}(\mathbf{r}, f)=4 D(\mathbf{r}, f)$, où $D$ est le coefficient de diffusion, les équations (17) et (18), portées dans (12), donnent $[2,4,5]$ :

$$
\mathbf{K}_{\mathrm{diff}}(\mathbf{r}, f)=4 q^{2} D(\mathbf{r}) \mathbf{D}(\mathbf{r}, f) .
$$

La comparaison directe des équations (19) et (15) donne une relation entre la température de bruit et le coefficient de diffusion :

$$
\begin{aligned}
& k_{\mathrm{B}} T_{n \alpha}(\mathbf{r}, f) / q= \\
& \quad=q n(r) D_{\alpha \alpha}(\mathbf{r}, f) / \operatorname{Re}\left\{\sigma_{\alpha \alpha}(\mathbf{r}, f)\right\} .
\end{aligned}
$$

Cette relation est valable même en régime de charge d'espace. Lorsque le champ électrique est uniforme, l'équation (20) donne une rèlation entre $T_{n}, D$, et la mobilité $\mu[6,7]$. Si de plus on est en régime ohmique, on retrouve la relation d'Einstein. Il a été démontré [9] que le coefficient de diffusion, défini comme ici à partir du bruit, était identique au coefficient de diffusion défini à partir de l'étalement d'un paquet de porteurs. Ainsi les mesures de bruit et de temps de vol, basées sur des principes totalement différents, donnent le même coefficient de diffusion.

3.4 BRUIT DE GÉNÉRATION-RECOMBINAISON (GR). - Le bruit G-R est dû aux fluctuations de densité de porteurs. L'équation (16) peut être exprimée à l'aide du nombre $\Delta N(\mathbf{r})$ de porteurs situés dans la tranche $\Delta A . \Delta x$ :

$$
I=q v(\mathbf{r}) \Delta N(\mathbf{r}) / \Delta x .
$$

En négligeant la contribution due aux fluctuations de vitesse, on obtient :

$$
S_{\mathrm{I}}(\mathbf{r}, f)=q^{2} v^{2}(\mathbf{r}) S_{\mathrm{N}}(\mathbf{r}, f) /(\Delta x)^{2}
$$

c'est-à-dire, d'après (21) :

$$
S_{\mathrm{I}}(\mathbf{r}, f)=j^{2}(\mathbf{r}) \Delta A^{2} S_{\mathrm{N}}(\mathbf{r}, f) / \Delta N^{2}(\mathbf{r}) .
$$

Cette relation, portée dans l'équation (12), donne :

$$
K(\mathbf{r}, f)=j^{2} \Delta A \Delta x S_{\mathrm{N}}(\mathbf{r}, f) / \Delta N^{2}(\mathbf{r}) .
$$

Pour du bruit G-R impliquant seulement deux niveaux [10] :

$$
S_{\mathrm{N}}=4 \tau(\operatorname{var} \Delta N) /\left(1+\omega^{2} \tau^{2}\right)
$$

et, à cause du caractère quasi Poissonien des fluctuations, var $N=\alpha \Delta N$. Ceci donne, pour l'équation (23) :

$$
K_{\mathrm{GR}}(\mathbf{r}, f)=\frac{j^{2}(\mathbf{r})}{n(\mathbf{r})} \frac{4 \alpha(\mathbf{r}) \tau(\mathbf{r})}{\left[1+\omega^{2} \tau^{2}(\mathbf{r})\right]}
$$

L'expression (24) est analogue à celle obtenue pour le bruit G-R thermique [10], à ceci près que d'une part $j(\mathbf{r})$ n'est pas nécessairement linéaire par rapport au champ électrique, d'autre part que $\alpha(\mathbf{r})$ et $\tau(\mathbf{r})$ peuvent dépendre du champ électrique local de polarisation. Une telle situation se présente, par exemple, dans Si-n à $77 \mathrm{~K}$ : alors, à l'équilibre thermique, une partie seulement des donneurs est ionisée ; lorsqu'un champ électrique est appliqué, une certaine fraction des impuretés neutres est 
ionisée, par effet Poole Frenkel et par impact ionisation, produisant du bruit G-R de porteurs chauds, puisque cette fraction dépend du champ, ainsi que $\alpha$ et $\tau$ [11] (voir $\S 4$ ci-dessous).

3.5 BRUIT EN $1 / f$. - En dépit des efforts prodigués par quelques douzaines de chercheurs depuis quelques douzaines d'années (voir les comptes rendus de chaque conférence sur le bruit et sur le bruit en $1 / f)$, il n'existe aucune théorie satisfaisante du bruit en $1 / f$. La plus ancienne, la plus simple, et peutêtre la plus réaliste, consiste à représenter le bruit en $1 / f$ comme la somme de bruits G-R produits par des sous-bandes d'impuretés ou de défauts, avec une distribution continue de temps de relaxation. Par suite, on peut déduire de l'équation (24) que la source de bruit de volume en $1 / f$ s'exprime par :

$$
K_{\mathrm{vol} 1 / f}(\mathbf{r}, f)=\frac{j^{2}(\mathbf{r})}{n(\mathbf{r})} \frac{\alpha_{\mathrm{H}}(\mathbf{r})}{f^{\gamma}}
$$

où $\alpha_{H}$ est appelée la constante de Hooge [12], qui est en fait un paramètre ajustable, pouvant varier [12] de quatre ordres de grandeur. Le facteur $\gamma$ est de l'ordre de 1 , généralement dans la fourchette 0,8 à 1,2 .

Très souvent, la technologie de croissance des matériaux est assez satisfaisante, de sorte qu'un tel bruit de volume est négligeable aux fréquences usuelles. Cependant, la technologie des surfaces et des interfaces est beaucoup plus mal maîtrisée, et des sources de bruit importantes en $1 / f$ peuvent être présentes, localisées sur une équipotentielle d'abscisse $x_{0}$. La source de bruit s'exprime alors par :

$$
K_{\text {surf } 1 / f}(x, f)=\lambda \frac{j^{2}(x)}{n(x)} \frac{\delta\left(x-x_{0}\right)}{f} .
$$

\section{Bruit dans les matériaux semiconducteurs.}

Il a été noté ci-dessus, que la mesure de la température de bruit et de la conductivité différentielle, permettait d'obtenir la source locale de bruit, dans un champ électrique uniforme. Une analyse du bruit dans une large gamme de fréquences permet d'obtenir des renseignements détaillés sur certains paramètres de transport, tels que le coefficient de diffusion $D(E)$, la densité de porteurs libres $n(E)$, la mobilité $\mu(E)$, la durée de vie $\tau(E)$, etc., bien sûr lorsque les conditions expérimentales sont favorables.

Ainsi, la figure 4 montre des résultats expérimentaux de mesure de température de bruit dans InP à $300 \mathrm{~K}$, entre $220 \mathrm{MHz}$ et $10 \mathrm{GHz}$, à différents champs électriques. De toute évidence, deux sources de bruit sont présentes: le bruit de diffusion, responsable du plateau à haute fréquence, et un bruit G-R correspondant au plateau en basse fréquence. Le courant du bruit s'écrit alors, d'après les équations (19), (24) et (12) :

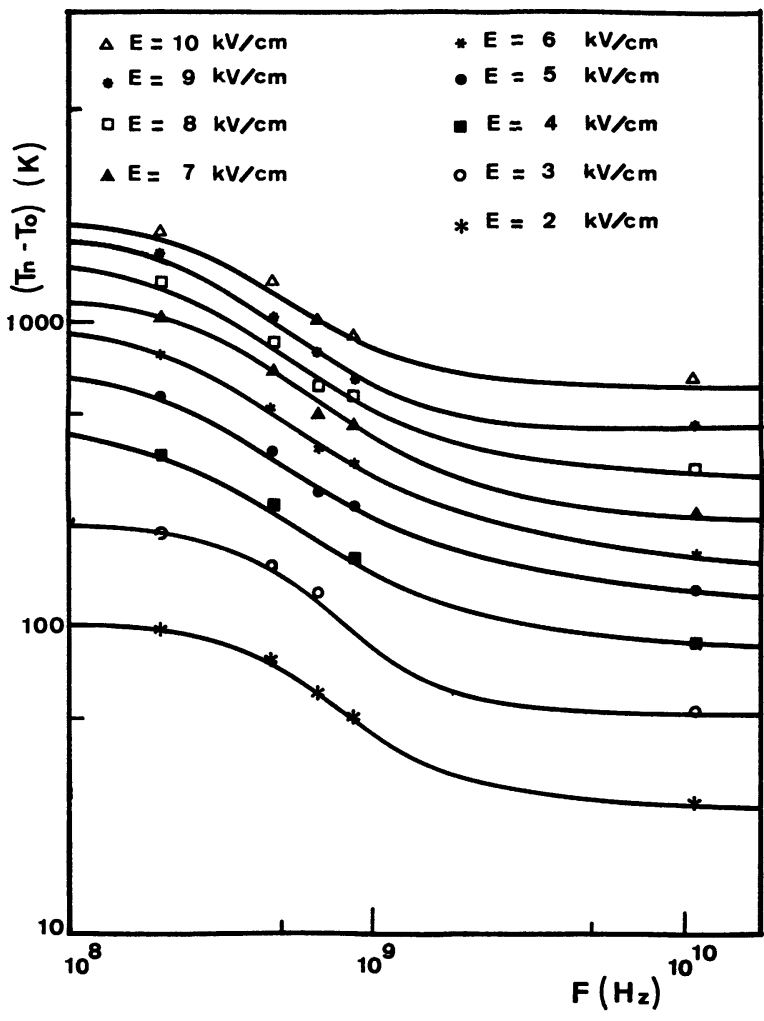

Fig. 4. - Température de bruit en excès, en fonction de la fréquence, pour différentes intensités du champ électrique, dans InP de type $\mathrm{n}$, à $300 \mathrm{~K}, N_{\mathrm{D}}=2,7 \times 10^{15} \mathrm{~cm}^{-3}$.

[Excess noise temperature versus frequency, at various electric field intensities, in n-type $\mathrm{InP}$, at $300 \mathrm{~K}, N_{\mathrm{D}}=$ $2.7 \times 10^{15} \mathrm{~cm}^{-3}$.]

$$
\begin{aligned}
& S_{\mathrm{I}}(f)=4 q^{2} n(E) D(E)(A / L)+ \\
& \quad+\frac{j^{2}}{n(E)} \frac{4 \alpha \tau}{\left(1+\omega^{2} \tau^{2}\right)} \frac{A}{L} .
\end{aligned}
$$

$\mathrm{Si}$ on suppose que le bruit G-R fait intervenir seulement deux niveaux, la bande de conduction et un niveau piège, on peut montrer [1] que :

$$
\alpha=(1-u) /(2-u) \text { avec } u=n / N_{\mathrm{D}} .
$$

Alors, l'équation (27) dépend seulement de 3 paramètres inconnus : $u(E), D(E)$, et $\tau(E)$. A un champ donné, il est aisé d'ajuster, par la méthode des moindres carrés, ces trois paramètres aux valeurs expérimentales (traits pleins de la Fig. 4), et par suite de déterminer les paramètres de transport $n(E), D(E)$ et $\tau(E)$, ce qui donne $\mu(E)$ puisque $\sigma(E)$ a été mesuré. La figure 5 montre les résultats obtenus pour $D(E)[14]$ : ceci a constitué la première détermination expérimentale du coefficient de diffusion dans InP. Des résultats similaires ont été obtenus dans Si-p [18], et dans Si-n à 77 K [17].

La figure 6 montre [17] clairement que dans Si-n à $77 \mathrm{~K}, N_{\mathrm{D}}=2,75 \times 10^{14} \mathrm{~cm}^{-3}$, le rapport $n(E) / N_{\mathrm{D}}$, égal à 0,75 à l'équilibre thermodynamique à $77 \mathrm{~K}$, 


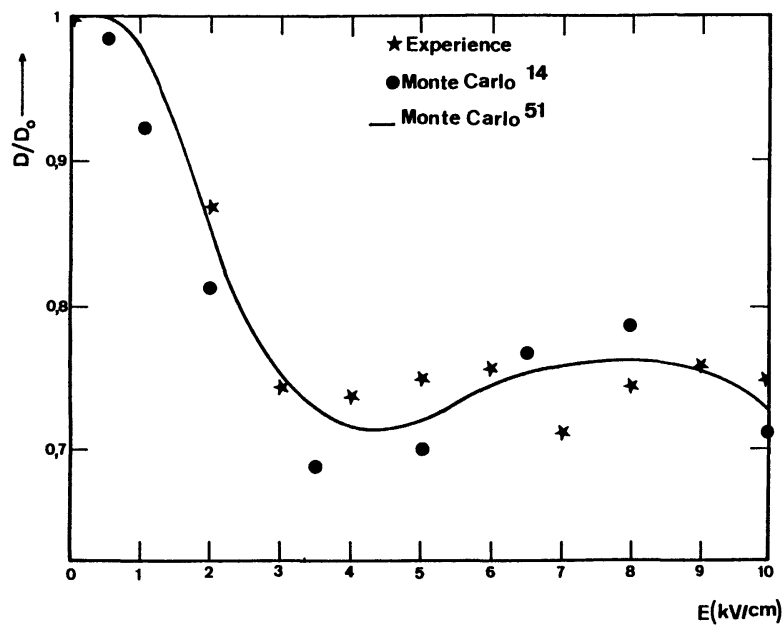

Fig. 5. - Coefficient de diffusion, en fonction du champ électrique, dans InP de type $\mathrm{n}, N_{\mathrm{D}}=2,7 \times 10^{15} \mathrm{~cm}^{-3}$, à $300 \mathrm{~K}$. Comparaison entre les résultats expérimentaux [14] (étoiles), et des simulations de Monte Carlo effectuées par Gasquet et al. [14] (cercles pleins), et par Hill et al. [51] (trait continu). La valeur ohmique est $D_{0}=116 \mathrm{~cm}^{2} / \mathrm{s}$.

[Diffusion coefficient versus electric field in n-type InP, $N_{\mathrm{D}}=2.7 \times 10^{15} \mathrm{~cm}^{-3}$, at $300 \mathrm{~K}$. Comparison between experimental results [14] (stars), and Monte Carlo simulations performed by Gasquet et al. [14] (dots), and by Hill et al. [51] (full line). The ohmic value is $D_{0}=116 \mathrm{~cm}^{2} / \mathrm{s}$.]

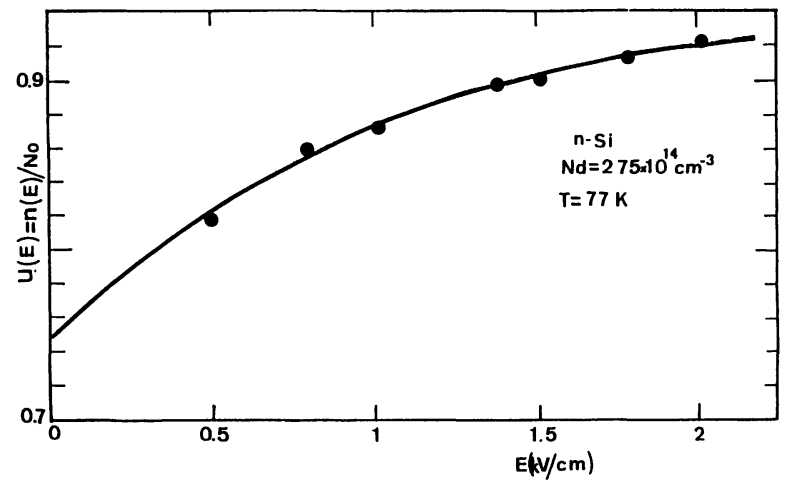

Fig. 6. - Variation de la densité d'électrons libres en fonction du champ électrique, dans Si-n à $77 \mathrm{~K}, N_{\mathrm{D}}=$ $2,75 \times 10^{14} \mathrm{~cm}^{-3}$, d'après [17].

[Variation of the density of free carriers, versus the electric field intensity, in n-type $\mathrm{Si}$, at $77 \mathrm{~K}, N_{\mathrm{D}}=2.75 \times$ $10^{14} \mathrm{~cm}^{-3}$, taken from [17].]

croît régulièrement avec le champ électrique, par effet Poole Frenkel ou par impact ionisation, pour atteindre 0,9 à $2 \mathrm{kV} / \mathrm{cm}$. La figure 7 montre la variation de $D(E)$.

La présence de bruit en $1 / f$ est en général le révélateur de problèmes technologiques : la figure 8 montre les bruits de deux contacts ohmiques, de même résistivité de contact. On voit qu'un des échantillons présente un bruit en $1 / f$ en dessous de

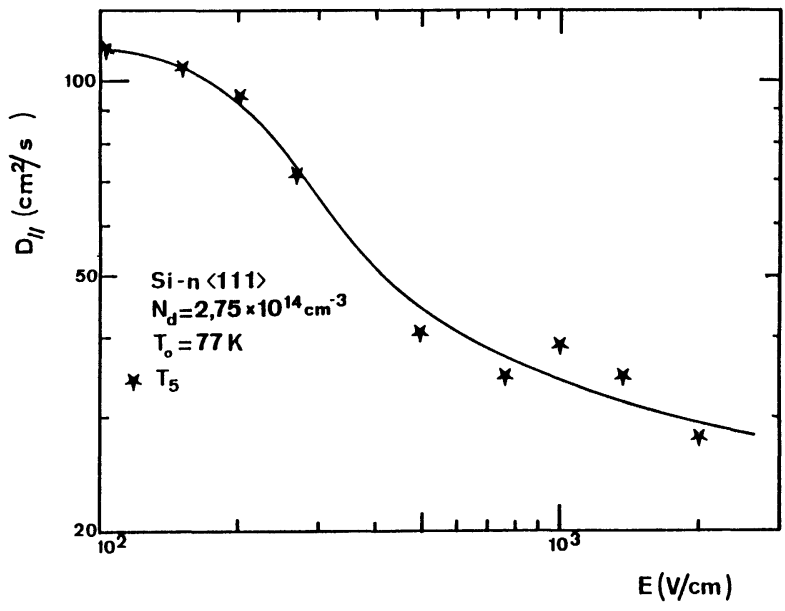

Fig. 7. - Variation du coefficient de diffusion en fonction du champ électrique, dans Si-n à $77 \mathrm{~K}, N_{\mathrm{D}}=2,75 \times$ $10^{14} \mathrm{~cm}^{-3}$, d'après [17].

[Variation of the diffusion coefficient, versus the electric field intensity, in n-type $\mathrm{Si}$, at $77 \mathrm{~K}, N_{\mathrm{D}}=2.75 \times$ $10^{14} \mathrm{~cm}^{-3}$, taken from [17].]

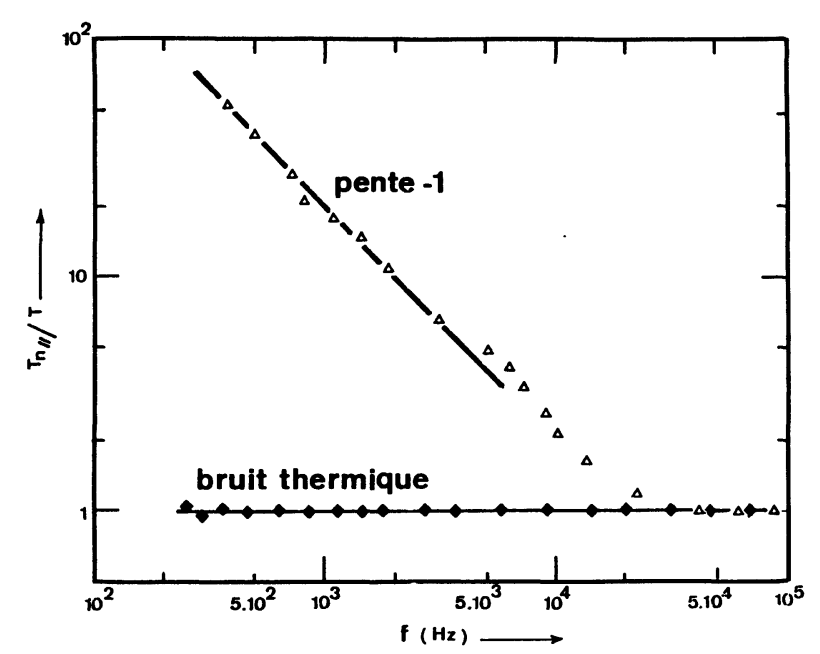

Fig. 8. - Bruit en $1 / f$ de contacts ohmiques d'eutectique $\mathrm{Ag}-\mathrm{Si}$ sur $\mathrm{Si}-\mathrm{n}$, de deux échantillons de même résistivité de contact $\left(\rho=6 \Omega \mathrm{cm}, \rho_{\mathrm{c}}=10 \Omega \mathrm{mm}^{2}, j=0.66 \mathrm{~A} / \mathrm{cm}^{2}\right.$, $T=300 \mathrm{~K})$, d'après [49].

$[1 / f$ noise of ohmic contacts obtained by Ag-Si eutectic formation on n-type $\mathrm{Si}$, for two samples with the same contact resistivity $\left(\rho=6 \Omega \mathrm{cm}, \rho_{\mathrm{c}}=10 \Omega \mathrm{mm}^{2}, j=\right.$ $\left.0,66 \mathrm{~A} / \mathrm{cm}^{2}, T=300 \mathrm{~K}\right)$, taken from [49].]

$20 \mathrm{kHz}$, alors que le second présente un bruit thermique jusqu'en dessous de $200 \mathrm{~Hz}$. Un tel bruit en $1 / f$ est en général dû à des microplasmas [52].

5. Bruit dans les composants : méthode du champ d'impédances.

5.1 FORMULATION DE LA MÉTHODE. — La méthode du champ d'impédances a été introduite pour la 
première fois par Shockley et al. [15] pour le bruit de diffusion dans le cas de composants unidimensionnels. Elle a par la suite été étendue au bruit G$\mathbf{R}[16]$, puis au cas tridimensionnel [2, 4] dans sa formulation dite du transfert d'impédance, qui a été ensuite généralisée de manière indépendante de la variable électrique employée [3]. Les extensions tridimensionnelles n'étant pas encore utilisées, et posant par ailleurs des problèmes fondamentaux, je donnerai ici une formulation unidimensionnelle simple mais un peu plus générale que celle donnée initialement par Shockley et al. [15].

Considérons (Fig. 9) un composant polarisé en continu entre les électrodes $\mathbf{M}$ et $\mathbf{N}$. Les électrodes de mesure sont $\mathbf{M}^{\prime}$ et $\mathrm{N}^{\prime}$ : on y mesure les variations ou les fluctuations autour du point de polarisation, à la fréquence $f$. On a tracé une ligne de courant alternatif, à la fréquence $f$, entre $\mathrm{M}^{\prime}$ et $\mathrm{N}^{\prime}$, ainsi que quelques équipotentielles. Les circuits de polarisation et de mesure doivent évidemment être découplés. Soit $x$ l'abscisse curviligne d'un point de la ligne de courant de longueur $L$. La surface équipotentielle passant par $x$ est au potentiel alternatif $V(x)$, lorsque l'électrode $\mathrm{N}^{\prime}$ est, par rapport à $\mathbf{M}^{\prime}$, au potentiel $V(L)$.

On considère trois états d'équilibre. Dans chacun, $\delta I(x)$ représente la valeur complexe d'un courant, à la fréquence $f$, suffisamment petit pour être en régime de petit signal autour du point de polarisation :

- $1^{\text {er }}$ état : $\delta I(x)$ est injecté entre $M^{\prime}$ et l'équipotentielle, supposée métallisée, passant par le point $x$. Le système est alors un quadripôle, dont l'entrée est située entre les points $\mathrm{M}^{\prime}$ et $x$, et la sortie entre les électrodes $\mathbf{M}^{\prime}$ et $\mathbf{N}^{\prime}$. Il apparaît alors en sortie, entre

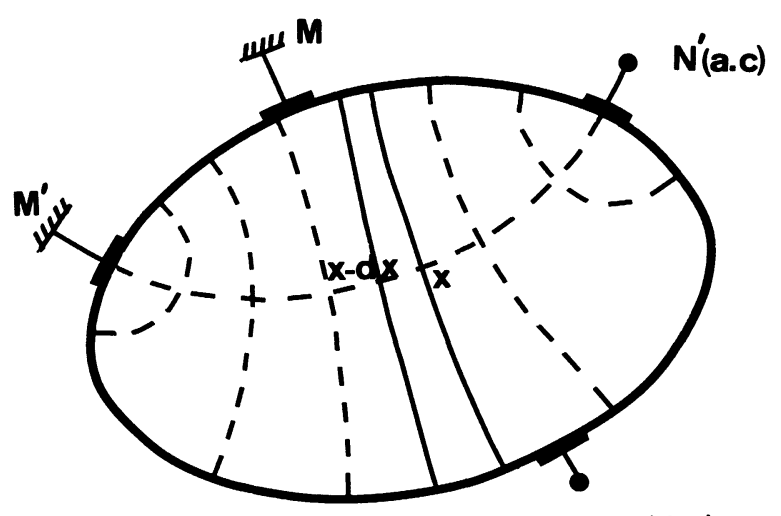

$\mathbf{N}$ (d.c)
Fig. 9. - Champ d'impédances dans un composant, polarisé en continu entre $M$ et $N$, et en alternatif entre $\mathbf{M}^{\prime}$ et $\mathrm{N}^{\prime}$. On a tracé quelques équipotentielles en alternatif.

[Impedance field method for a device with $\mathrm{M}$ and $\mathrm{N}$ as d.c. electrodes, and $\mathrm{M}^{\prime}$ and $\mathrm{N}^{\prime}$ as a.c. electrodes. A few a.c. equipotential surfaces have been drawn.]
$\mathrm{M}^{\prime}$ et $\mathrm{N}^{\prime}$, une tension $\Delta V_{+}$, proportionnelle à $\delta I(x):$

$$
\Delta V_{+}=Z(x, f) \delta I(x)
$$

où $Z(x, f)$ est homogène à une impédance.

- $2^{\mathrm{e}}$ état : le même courant $\delta I(x)$ est retiré, entre $\mathbf{M}^{\prime}$ et l'équipotentielle passant par le point $x-\mathrm{d} x$. Il apparaît alors, entre $\mathrm{M}^{\prime}$ et $\mathrm{N}^{\prime}$, une tension $\Delta V_{-}$, proportionnelle à $\delta I(x)$ :

$$
\Delta V_{-}=Z(x-\mathrm{d} x, f) \delta I(x) .
$$

- $3^{\mathrm{e}}$ état : $\delta I(x)$ est injecté à l'équipotentielle passant par $x$, et retiré à l'équipotentielle passant par $x-\mathrm{d} x$ (Fig. 9) : cet état est la superposition des deux états d'équilibre précédents. La tension entre $\mathrm{M}^{\prime}$ et $\mathrm{N}^{\prime}$ vaut alors :

$$
\begin{aligned}
\delta V(x)=\Delta V_{+}- & \Delta V_{-}= \\
& =[Z(x, f)-Z(x-\mathrm{d} x, f)] \delta I(x)
\end{aligned}
$$

soit

$$
\delta V(x)=\nabla Z(x, f) \delta I(x) \mathrm{d} x
$$

$\nabla Z(x, f)$ est le champ d'impédance.

La signification physique de l'équation (30) est simple : le composant, en ce qui concerne le petit signal alternatif, est un quadripôle dont l'entrée, constituée par les deux équipotentielles passant par les points $x$ et $x-\mathrm{d} x$, est attaquée par un courant alternatif de valeur complexe $\delta I(x)$ à la fréquence $f$. La sortie est constituée par les électrodes $\mathbf{M}^{\prime}$ et $\mathrm{N}^{\prime}$. Il apparaît entre $\mathrm{M}^{\prime}$ et $\mathrm{N}^{\prime}$ une tension $\delta V(x)$, proportionnelle à $\delta I(x)$, la fonction de transfert étant une impédance différentielle, proportionnelle à $\mathrm{d} x$.

La tension totale, entre $\mathrm{M}^{\prime}$ et $\mathrm{N}^{\prime}$, est la somme des tensions dues à tous les courants apparus dans toutes les tranches $\mathrm{d} x$. On a donc:

$$
\delta V=\int_{0}^{L} \nabla Z(x, f) \delta I(x) \mathrm{d} x .
$$

Dans les dipôles, $\delta I(x)$ sera constant, mais dans les quadripôles, $\delta I(x)$ peut effectivement dépendre de $x$ [35].

Si maintenant $\delta I(x)$ est un courant aléatoire à la fréquence $f, \delta V$ est une tension aléatoire, dont la densité spectrale est obtenue en prenant la moyenne de $\delta V . \delta V^{*}$ (cf. Eq. (1)). On a :

$$
\begin{aligned}
\delta V . \delta V^{*}=\int_{0}^{L} \int_{0}^{L} & \nabla Z(x, f) \nabla Z^{*}\left(x^{\prime}, f\right) \times \\
& \times \delta I(x) \delta I^{*}\left(x^{\prime}\right) \mathrm{d} x \mathrm{~d} x^{\prime} .
\end{aligned}
$$

En prenant la moyenne des deux membres, et en multipliant par la bande $\Delta f$ (cf. Eq. (i)), il vient :

$$
\begin{aligned}
S_{V}(f)=\int_{0}^{L} \int_{0}^{L} \nabla Z(x, f) & S_{\mathrm{I}}\left(x, x^{\prime}, f\right) \times \\
& \times \nabla Z^{*}\left(x^{\prime}, f\right) \mathrm{d} x \mathrm{~d} x^{\prime} .
\end{aligned}
$$


Ainsi qu'il a déjà été dit, on admet généralement que les sources de bruit en $x^{\prime} \neq x$ sont décorrélées. En portant l'équation (11) dans l'équation (33), il vient, après intégration sur $x^{\prime}$ :

$$
S_{\mathrm{V}}(f)=\int_{0}^{L} A(x) K(x, f)|\nabla Z(x, f)|^{2} \mathrm{~d} x
$$

où la section a été désignée par $A(x)$ au lieu de $\Delta A(x) . A(x)$ est l'aire de la surface équipotentielle passant par $x$. La relation (34) est celle initialement donnée par Shockley [15].

5.2 UTILISATION DU CHAMP ÉLECTRIQUe LOCAL. - Dans un certain nombre de problèmes, la variable électrique la plus commode est le champ électrique. La méthode de détermination du champ d'impédances dans ce cas, a été donnée par Van Vliet et al. [2] qui l'ont appelée méthode du transfert d'impédances. A une dimension, la procédure est la suivante :

a) On écrit les équations de transport, et on élimine les variables intermédiaires, de façon à obtenir une relation entre le champ local $E(x)$ et le courant $I$. Cette relation est en général non linéaire, dès qu'on est en régime de charge d'espace ou de porteurs chauds. Elle s'écrit :

$$
f(E, x)=I \text {. }
$$

La résolution de cette équation, en régime stationnaire, donne la relation entre le champ local $E_{0}(x)$, et le courant de polarisation $I_{0}$. Ceci donne le point de polarisation $\left(I_{0}, V_{0}\right)$.

b) On superpose à $I_{0}$ un petit courant alternatif $\delta I \exp j \omega t$, le champ local alternatif vaut $\delta E \exp j \omega t$. On pose donc, dans l'équation (35) :

$$
E=E_{0}(x)+\delta E(x, f) \exp j \omega t
$$

et

$$
I=I_{0}+\delta I(x, f) \exp j \omega t
$$

et on développe l'équation (35) en perturbations. Les termes d'ordre 0 redonnent le point de polarisation, les termes d'ordre 1 en $\delta I$ et $\delta E$ donnent une relation de la forme :

$$
\hat{L} \delta E(x, f)=\delta I(x, f)
$$

où $\hat{L}$ est par définition un opérateur linéaire.

c) La fonction de Green $z\left(x, x^{\prime}, f\right)$ de l'opérateur $\hat{L}$ est solution de l'équation :

$$
\hat{L} z\left(x, x^{\prime}, f\right)=\delta\left(x-x^{\prime}\right)
$$

où $\delta\left(x-x^{\prime}\right)$ est la fonction de Dirac.

d) $\delta E(x, f)$ est alors donné par:

$$
\delta E(x, f)=\int_{0}^{L} \mathrm{~d} x^{\prime} z\left(x, x^{\prime}, f\right) \delta I\left(x^{\prime}, f\right) .
$$

En intégrant de 0 à $L$, il vient :

$$
\delta V=-\int_{0}^{L} \mathrm{~d} x^{\prime} \delta I\left(x^{\prime}, f\right) \int_{0}^{L} \mathrm{~d} x z\left(x, x^{\prime}, f\right) .
$$

La comparaison des équations (39) et (31) montre que :

$$
\nabla Z\left(x^{\prime}, f\right)=-\int_{0}^{L} z\left(x, x^{\prime}, f\right) \mathrm{d} x .
$$

e) L'impédance différentielle à la fréquence $f$ est donnée par:

$$
\begin{aligned}
Z(f)=\int_{0}^{L} \nabla Z\left(x^{\prime}, f\right) \mathrm{d} x^{\prime}= \\
\quad=-\int_{0}^{L} \int_{0}^{L} z\left(x, x^{\prime}, f\right) \mathrm{d} x \mathrm{~d} x^{\prime}
\end{aligned}
$$

et la tension de bruit est donnée par l'équation (34).

Exemple: diode à injection en simple charge d'espace.

Considérons $[2,30-32]$ une diode $\mathrm{p}^{+}-\mathrm{p}-\mathrm{p}^{+}$, où les porteurs dans la diode proviennent de ceux injectés à une électrode.

a) Les équations de transport sont :

$$
\begin{aligned}
& I=q p v A+\varepsilon A \partial E / \partial t \\
& \partial E / \partial x=(q / \varepsilon)\left(p-N_{\mathrm{A}}\right) \simeq(q / \varepsilon) p
\end{aligned}
$$

où la vitesse de dérive $v=v(E)$ peut ne pas être proportionnelle à $E, A$ est la surface de la diode. L'élimination de $p$ entre ces deux équations donne (cf. Eq. (35)) :

$$
I=\varepsilon A v(\partial E / \partial x)+\varepsilon A(\partial E / \partial t) .
$$

Le point de polarisation est obtenu en annulant les dérivées partielles par rapport au temps :

$$
\begin{aligned}
& I_{0}=q p_{0} v_{0} A \\
& \mathrm{~d} E_{0} / \mathrm{d} x=(q / \varepsilon) p_{0}
\end{aligned}
$$

c'est-à-dire :

$$
I_{0}=\varepsilon A v_{0}\left(\mathrm{~d} E_{0} / \mathrm{d} x\right) .
$$

Lorsque la loi de variation $v(E)$ est connue, l'intégration de l'équation (45) donne le profil de champ $x\left(E_{0}\right)$, donc $E_{0}(x)$ :

$$
x I_{0}=\varepsilon A \int_{0}^{E_{0}} v_{0}(E) \mathrm{d} E
$$

L'une des équations (44) donne alors $p_{0}$. Tirant $\mathrm{d} x$ de l'équation (45), il vient :

$V_{0}=-\int_{0}^{x} E_{0} \mathrm{~d} x=-\left(\varepsilon A / I_{0}\right) \int_{0}^{E_{0}} E v_{0}(E) \mathrm{d} E$. 
b) Pour obtenir le régime dynamique et le bruit, on pose :

$I=I_{0}+\delta I \exp (j \omega t)$ et $E=E_{0}+\delta E \exp (j \omega t)$.

Ces valeurs, portées dans l'équation (43), donnent pour les termes d'ordre un (cf. Eq. (36)) :

$$
\begin{aligned}
& \varepsilon A v_{0}(\mathrm{~d} \delta E / \mathrm{d} x)+ \\
& +\left[\varepsilon A\left(\mathrm{~d} v_{0} / \mathrm{d} x\right)+j \omega \varepsilon A\right] \delta E=\delta I .
\end{aligned}
$$

c) La fonction de Green est solution de (cf. Eq. (37)) :

$$
\begin{array}{r}
\varepsilon A v_{0}(x)\left(\mathrm{d} z\left(x, x^{\prime}, f\right) / \mathrm{d} x\right)+\left[\varepsilon A\left(\mathrm{~d} v_{0} / \mathrm{d} x\right)+\right. \\
+j \omega \varepsilon A] z\left(x, x^{\prime}, f\right)=\delta\left(x-x^{\prime}\right)
\end{array}
$$

On détermine d'abord la solution de cette équation sans second membre, en utilisant le fait que, d'après l'équation (45), $\mathrm{d} x=\left(\varepsilon A v_{0} / I_{0}\right) \mathrm{d} E_{0}$. La méthode de la variation de la constante donne alors $z\left(x, x^{\prime}, f\right)$, après avoir annulé la constante d'intégration, car

$$
z\left(x=0, x^{\prime}, f\right)=0
$$

puisque $\delta E(x=0)=0$. Par suite :

$$
\begin{aligned}
z\left(x, x^{\prime}, f\right) & =\left[H\left(x-x^{\prime}\right) /\left(\varepsilon A v_{0}(x)\right)\right] \times \\
& \times \exp \left\{j \omega \varepsilon A\left[E_{0}\left(x^{\prime}\right)-E_{0}(x)\right] / I_{0}\right\}
\end{aligned}
$$

où $H\left(x-x^{\prime}\right)$ est la fonction échelon, $H=1$ si $x \geqslant x^{\prime}, H=0$ si $x<x^{\prime}$.

d) Conformément à l'équation (40), l'intégration sur $x$ de l'équation (50), compte tenu ici aussi de l'équation (45), donne :

$$
\begin{array}{r}
\nabla Z\left(x^{\prime}\right)=\left[\exp \left\{j \omega \varepsilon A\left[E_{0}\left(x^{\prime}\right)-E_{0}(L)\right] / I_{0}\right\}-\right. \\
-1] /(j \omega \varepsilon A) .
\end{array}
$$

e) Dans le cas du bruit de diffusion, on obtient (cf. Eqs. (19) et (45)) :

$K(x, f)=4 q^{2} p D\left(E_{0}\right)=4 q \varepsilon D\left(E_{0}\right)\left(\mathrm{d} E_{0} / \mathrm{d} x\right)$.

Les équations (52) et (51), portées dans l'équation (34), donnent finalement :

$$
\begin{aligned}
S_{\mathrm{V}}(f)=( & \left.16 q / \omega^{2} \varepsilon A\right) \int_{0}^{E_{0}(L)} \mathrm{d} E D(E) \times \\
& \times \sin ^{2}\left\{\omega \varepsilon A\left[E-E_{0}(L)\right] /\left(2 I_{0}\right)\right\}
\end{aligned}
$$

En basse fréquence, $f \rightarrow 0$, on obtient [31] :

$$
\begin{aligned}
S_{\mathrm{V}}(f=0)=\left(4 A q \varepsilon / I_{0}^{2}\right) & \int_{0}^{E_{0}(L)} D(E) \times \\
& \times\left[E-E_{0}(L)\right]^{2} \mathrm{~d} E .
\end{aligned}
$$

Cet exemple montre que la connaissance des variations de $v$ et $D$ avec $E$, permet d'obtenir le régime stationnaire, le régime dynamique, ainsi que le bruit. En particulier, la contribution, au bruit total, du bruit produit par la tranche $\mathrm{d} x$ à l'abscisse $x$, où règne un champ $E_{0}$ à $\mathrm{d} E_{0}$ près, vaut $D\left(E_{0}\right)\left[E_{0}-E_{0}(L)\right]^{2} \mathrm{~d} E_{0}$ : on est donc en mesure de chiffrer la contribution au bruit total, apportée par chaque tranche.

5.3 Utilisation du potentiel local. - Dans certains cas, la variable électrique la plus commode n'est pas le champ électrique local $E(x)$, mais le potentiel local $V(x)$. Dans ce cas $[34,35]$, on applique comme précédemment une petite tension alternative aux électrodes $\mathbf{M}^{\prime}$ et $\mathrm{N}^{\prime}$ (cf. Fig. 9), superposée à la tension continue de polarisation. Soit $\delta V(x) \exp (j \omega t)$ le potentiel alternatif de la surface équipotentielle, de section $A(x)$, coupant une ligne de courant de longueur $L$, à l'abscisse curviligne $x$, et soit $\delta I(x) \exp (j \omega t)$ le courant alternatif à travers cette surface équipotentielle. Comme précédemment, $\delta V$ et $\delta I$ sont obtenus en écrivant les équations de transport, ce qui donne, au premier ordre :

$$
\mathcal{L} \delta V(x)=\delta I(x)
$$

où $\mathcal{L}$ est un opérateur linéaire. La fonction de Green $\mathcal{Z}\left(x, x^{\prime}, f\right)$ de l'équation (55) est solution de :

$$
\mathfrak{L} \mathfrak{3}\left(x, x^{\prime}, f\right)=\delta\left(x-x^{\prime}\right)
$$

et donne :

$$
\delta V(x)=\int_{0}^{L} 3\left(x, x^{\prime}, f\right) \delta I\left(x^{\prime}\right) \mathrm{d} x^{\prime}
$$

c'est-à-dire, lorsque $x=L$ :

$$
\delta V(x=L)=\int_{0}^{L} 3\left(L, x^{\prime}, f\right) \delta I\left(x^{\prime}\right) \mathrm{d} x^{\prime} .
$$

En comparant les équations (58) et (31), on obtient immédiatement :

$$
\nabla Z\left(x^{\prime}, f\right)=3\left(L, x^{\prime}, f\right) .
$$

\section{Exemple: Transistor à Effet de Champ (FET).}

Considérons un FET à canal n (FET à jonction, MES-FET ou MOS-FET). Pour des valeurs données $V_{\mathrm{G}}$ et $V_{\mathrm{D}}=V(L)$ des tensions grille et drain, par rapport à la source, la section $A(x)$ du canal, à la distance $x$ de la source, est une fonction du potentiel local $V(x)$ sous la grille, $A$ pouvant être constant dans les régions d'accès source et drain (ceci n'est pas toujours vrai, à cause par exemple de l'extension de la charge d'espace de la jonction canal substrat, etc...). En supposant que les surfaces équipotentielles ont un rayon de courbure grand devant la section du canal, une approximation unidimensionnelle est acceptable. On peut donc écrire $A(x)=A(V(x))$, 
ce qui incite à prendre $V(x)$ comme variable électrique fondamentale.

En négligeant les courants de diffusion et de déplacement, les équations de transport s'écrivent :

$$
\left\{\begin{array}{l}
I=q n v A \\
\mathrm{~d}(E A) / \mathrm{d} x=q A\left(N_{\mathrm{D}}-n\right) / \varepsilon .
\end{array}\right.
$$

L'équation (60b) est l'équation de Poisson, intégrée sur le volume d'une tranche du canal, comprise entre les surfaces équipotentielles $V(x)$ et $V(x+\mathrm{d} x)$ (on désigne ici par « canal » toute la zone active comprise entre la source et le drain). La charge d'espace étant faible, sera négligée. On a alors $n \simeq N_{\mathrm{D}}$, et les équations (60a) et (60b) deviennent :

$$
\left\{\begin{array}{l}
I=q N_{\mathrm{D}} v A \\
E(x) A(V(x)) \simeq E(0) A(0)
\end{array}\right.
$$

En prenant $E \leqslant 0, v \geqslant 0$, et $I \geqslant 0$, la loi de variation de $v$ avec $E$ pourra s'approcher par:

$$
v=-\mu_{0} E /\left(1-\left(E / E_{\mathrm{c}}\right)\right) \text {. }
$$

Cette relation, portée dans (61a), donne :

$$
I\left(1-\left(E / E_{\mathrm{c}}\right)\right)=-q N_{\mathrm{D}} \mu_{0} E A(V) .
$$

Ceci donne le point de polarisation et les quantités associées $I_{0}, E_{0}(x), V_{0}(x)$. Pour obtenir le bruit, on pose :

$$
\begin{aligned}
I & =I_{0}+\delta I \exp (j \omega t), \\
E & =E_{0}+\delta E \exp (j \omega t), \\
V & =V_{0}+\delta V \exp (j \omega t), \\
\delta E & =-\mathrm{d} \delta V / \mathrm{d} x .
\end{aligned}
$$

Ces relations, portées dans l'équation (63), donnent au premier ordre :

$$
\left\{\begin{array}{l}
a(x) \mathrm{d} \delta V / \mathrm{d} x-b(x) \delta V(x)=\delta I(x) \\
a(x)=\left(q N_{\mathrm{D}} \mu_{0} A_{0}-\left(I_{0} / E_{\mathrm{c}}\right)\right) /\left(1-\left(E_{0} / E_{\mathrm{c}}\right)\right) \\
b(x)=-q N_{\mathrm{D}} \mu_{0} E_{0}(\mathrm{~d} A / \mathrm{d} V)_{0} /\left(1-\left(E_{0} / E_{\mathrm{c}}\right)\right)
\end{array}\right.
$$

Ceci est l'équation (55). La fonction de Green $3\left(x, x^{\prime}, f\right)$ (cf. Eq. (56) est solution de :

$$
a(x) \mathrm{d} 3\left(x, x^{\prime}\right) / \mathrm{d} x+b(x) 3\left(x, x^{\prime}\right) .
$$

On voit qu'ici $\dot{3}\left(x, x^{\prime}\right)$ ne dépend pas de $f$. La solution de l'équation (65) est obtenue de la même manière que dans l'exemple précédent, et s'écrit :

$$
\begin{aligned}
& 3\left(x, x^{\prime}\right)=\left[H\left(x-x^{\prime}\right) / a\left(x^{\prime}\right)\right] \times \\
& \quad \times \exp \int_{x}^{x^{\prime}}(b(u) / a(u)) \mathrm{d} u
\end{aligned}
$$

L'intégrale dans l'équation (66) peut être calculée en utilisant le changement de variable : $-E_{0} \mathrm{~d} u=\mathrm{d} V$. En remarquant que $(\mathrm{d} A / \mathrm{d} V)_{0} \mathrm{~d} V=\mathrm{d} A_{0}$, l'équa- tion (66) s'intègre analytiquement, ce qui donne :

$\not{Z}\left(x, x^{\prime}\right)=H\left(x-x^{\prime}\right)\left(1-\left(E_{0}\left(x^{\prime}\right) / E_{\mathrm{c}}\right)\right)$

$$
\left(q N_{\mathrm{D}} \mu_{0} A_{0}(x)-\left(I_{0} / E_{\mathrm{c}}\right)\right)
$$

c'est-à-dire, en tenant compte de l'équation (63) :

$3\left(x, x^{\prime}\right)=-\left(E_{0}(x) / I_{0}\right)\left(1-\left(E_{0}\left(x^{\prime}\right) / E_{\mathrm{c}}\right)\right) \times$

$$
\times H\left(x-x^{\prime}\right)
$$

D'après l'équation (59), le champ d'impédance vaut :

$$
\nabla Z\left(x^{\prime}\right)=-\left(E_{0}(L) / I_{0}\right)\left(1-\left(E_{0}\left(x^{\prime}\right) / E_{\mathrm{c}}\right)\right)
$$

ce qui donne l'impédance différentielle, en intégrant sur $x$ :

$$
Z=-\left(E_{0}(L) / I_{0}\right)\left(L+\left(V(L) / E_{\mathrm{c}}\right)\right) .
$$

La tension de bruit est donnée par l'équation (34), et le courant de bruit vaut $S_{\mathrm{I}}=S_{\mathrm{V}} /|Z|^{2}$ :

$$
\begin{aligned}
S_{\mathrm{I}}= & L^{-2}\left[1+\left(V(L) / E_{\mathrm{c}} L\right)\right]^{-2} \times \\
& \times \int_{0}^{\mathrm{L}} K\left(x^{\prime}\right)\left(1-\left(E_{0}\left(x^{\prime}\right) / E_{\mathrm{c}}\right)\right)^{2} A\left(x^{\prime}\right) \mathrm{d} x^{\prime}
\end{aligned}
$$

Etude de quelques cas particuliers.

a) Bruit de diffusion dans les FETs : en utilisant les équations (19) et (20), où, d'après (62), $\sigma=$ $\mathrm{d} j_{0} / \mathrm{d} E_{0}=-q N_{\mathrm{D}} \mu_{0} /\left(1-E / E_{\mathrm{c}}\right)^{2}$, l'équation (71) devient :

$$
\begin{aligned}
S_{\mathrm{I}(\text { diff })=4 k_{\mathrm{B}} \mu_{0} q N_{\mathrm{D}}} & L^{-2}\left[1+\left(V(L) /\left(E_{\mathrm{c}} L\right)\right)\right]^{-2} \times \\
& \times \int_{0}^{L} T_{n}\left(x^{\prime}\right) A\left(x^{\prime}\right) \mathrm{d} x^{\prime} .
\end{aligned}
$$

Cette équation montre que, en première approximation, toutes les tranches du FET apportent une contribution comparable au bruit, puisque les tranches où $E$ est élevé, et donc où $T_{n}\left(x^{\prime}\right)$ est élevé, sont celles où $A\left(x^{\prime}\right)$ est petit.

b) Bruit G-R dans les FETs : il est obtenu en remplaçant, dans l'équation (71), $K\left(x^{\prime}\right)$ par son expression (24), et en tenant compte des équations (61a), (61b) et (62), ce qui donne :

$$
\begin{aligned}
S_{\mathrm{I}(\mathrm{G}-\mathrm{R})} 4 q \mu_{0} L^{-2} & {\left[1+\left(V(L) /\left(E_{\mathrm{c}} L\right)\right)\right]^{-2} \times } \\
& \times \alpha \tau\left(1+\omega^{2} \tau^{2}\right)^{-1} I_{0} V(L)
\end{aligned}
$$

On voit que, dans les FETs longs $\left(E_{\mathrm{c}} L \gg V(L)\right)$, le bruit G-R est proportionnel à la puissance continue dissipée $I_{0} V(L)$.

c) Bruit de volume en $1 / f$ dans les FETs : d'après l'équation (25), on l'obtient à partir du bruit G-R en remplaçant $4 \alpha \tau\left(1+\omega^{2} \tau^{2}\right)^{-1}$ par $\alpha_{\mathrm{H}} / f$, ce qui donne :

$$
\begin{aligned}
S_{\mathrm{I}(1 / f \text { vol })}=\alpha_{\mathrm{H}} q \mu_{0} L^{-2}[1+ & \left.\left(V(L) /\left(E_{\mathrm{c}} L\right)\right)\right]^{-2} \times \\
& \times f^{-1} I_{0} V(L)
\end{aligned}
$$


ce qui montre que, dans les FETs longs, le bruit de volume en $1 / f$ est lui aussi proportionnel à la puissance continue $I_{0} V(L)$.

d) Bruit de contact en $1 / f$ dans les FETs : il est obtenu en remplaçant, dans l'équation (71), $K\left(x^{\prime}\right)$ par (26), ce qui donne :

$$
\begin{aligned}
S_{\mathrm{I}(1 / f \text { contact })} & =\left[\lambda /\left(N_{\mathrm{D}} A(0)\right)\right] \times \\
\times & L^{-2}\left(1+V(L) /\left(E_{\mathrm{c}} L\right)\right)^{-2}\left(I_{0}^{2} / f\right)
\end{aligned}
$$

Cette relation montre que, contrairement au bruit en $1 / f$ de volume, le bruit en $1 / f$ de contact n'est pas proportionnel à la puissance continue $I_{0} V(L)$, mais à $I_{0}^{2}$. Son comportement est donc totalement différent du précédent en régime de saturation du transistor : dans ce cas, le bruit en $1 / f$ dû au contact reste constant, alors que celui en volume est proportionnel à la tension drain.

Une procédure similaire peut être appliquée pour calculer le courant de bruit induit dans la grille par les fluctuations dans le canal, ainsi que la corrélation entre les courants de bruit de grille et de drain [35]. De la connaissance des courants de bruit de drain et de grille, et de leur corrélation, on peut alors déduire à partir du schéma équivalent du transistor, son facteur de bruit, qui est en fait la grandeur intéressante pour l'ingénieur.

5.4 Simulation NUMÉRIQUe DiRecte. - Il est malheureusement impossible, en général, de calculer le champ d'impédances, et donc le bruit, en conduisant les calculs analytiques aussi loin qu'il n'a été fait dans les exemples précédents. Par conséquent, une technique numérique est nécessaire.

Une méthode a été appliquée, par le groupe de Lille, au MES-FET GaAs [20-22], au MES-FET double grille GaAs [23, 24], et au TEG-FET [24-27]. Cette méthode découle de l'équation (31): si on suppose que $\delta I(x)=1$ dans la tranche $\Delta x$, et est nul partout ailleurs, l'équation (31) donne : $\delta V=$ $\nabla Z(x, f) \Delta x$. La méthode est donc la suivante :

a) Ecrire les équations de transport.

b) Les linéariser, pour obtenir une relation entre la grandeur électrique locale (champ $\delta E(x)$ ou potentiel $\delta V(x)$ ), et le courant local $\delta I(x)$.

c) Discrétiser la longueur du composant.

d) Poser $\delta I\left(x_{j}\right)=1$ au point $x_{j}$, et 0 ailleurs, et calculer la différence de potentiel $\delta V_{j}$ aux électrodes. Le champ d'impédances $\nabla Z\left(x_{j}\right)$ vaut $\nabla Z_{j}=$ $\delta V_{j} / \Delta x_{j}$.

e) Calculer le bruit en utilisant l'équation (34).

Le formalisme est le suivant [33] : après avoir discrétisé la longueur $L$ du composant, l'équation (36) s'écrit, en chaque point $x_{i}$, puisque l'opérateur $\hat{L}$ est linéaire :

$$
\sum_{j} a_{i j} \delta E_{j}=\delta I_{i}, \quad \text { soit } \mathbf{A} \delta \mathbf{E}=\delta \mathbf{I} .
$$

Ce système linéaire est résolu, ce qui donne :

$$
\delta \mathbf{E}=\mathbf{Z} \delta \mathbf{I}, \text { avec } \mathbf{Z}=\mathbf{A}^{-1}
$$

c'est-à-dire :

$$
\delta E_{k}=\sum_{j} Z_{k j} \delta I_{j}
$$

Par ailleurs, l'équation (38), une fois discrétisée, s'écrit :

$$
\delta E_{k}=\sum_{j} z\left(x_{k}, x_{j}, f\right) \delta I_{j} \Delta x_{j} .
$$

La comparaison directe des équations (78) et (77) montre que la fonction de Green est :

$$
z\left(x_{k}, x_{j}, f\right)=Z_{k j} / \Delta x_{j} .
$$

De la même manière, la discrétisation de l'équation (40) donne :

$$
\nabla Z_{j}=-\sum_{k} z\left(x_{k}, x_{j}, f\right) \Delta x_{k}
$$

ce qui, par comparaison avec (79), donne :

$$
\nabla Z_{j}=-\sum_{k} Z_{k j} \Delta x_{k} / \Delta x_{j}
$$

Ainsi, la détermination de la matrice $\mathbf{Z}$, inverse de A, donne directement le champ d'impédances $\nabla Z_{j}$ en chaque point, et donc le bruit. Cette méthode a été aussi appliquée [33] aux diodes $\mathrm{p}^{+}-\mathrm{p}-\mathrm{p}^{+}$.

Une procédure tout à fait analogue peut être développée dans le cas où le potentiel local est la variable électrique choisie [36].

La méthode numérique exposée au début de ce paragraphe a aussi été utilisée par Brewitt-Taylor et al. [28]. La méthode du champ d'impédances peut même être employée sans écrire les équations de transport. Ceci a été fait dans un FET [29], considéré comme une ligne active constituée d'une chaîne de transistors élémentaires. Le bruit est alors considéré comme la somme des effets, au drain et à la grille, des bruits produits par les différentes tranches, où chaque tranche est un transistor élémentaire.

\section{Problèmes soulevés par les composants submicro- niques.}

Le comportement en bruit des composants submicroniques est différent de celui de composants plus longs, essentiellement pour deux raisons :

- Tout d'abord, certains paramètres « macroscopiques ", qui n'avaient pas d'effet dans les composants « longs », prennent ici une importance inattendue.

- Surtout, de nouveaux phénomènes interviennent, qui ne sont en général pas pris en considération dans les composants longs. Certains de ces problè- 
mes ne sont pas encore résolus, et ont seulement été formulés très récemment.

\subsection{COMPORTEMENT INHABITUEL DE PARAMÈTRES} «MACROSCOPIQUES ». - Considérons par exemple le courant de bruit de drain d'un FET, dans le cas du bruit G-R, donné par l'équation (73). Dans le silicium, $E_{\mathrm{c}} \simeq 10 \mathrm{kV} / \mathrm{cm}$ à $300 \mathrm{~K}$, de sorte que, en régime de saturation, avec une tension de drain $V_{\mathrm{D}}=V(L)=5 \mathrm{~V}$, deux cas limites peuvent être examinés :

- Dans les composants longs, par exemple un FET à jonction, de longueur $L \simeq 20 \mu \mathrm{m}$ entre source et drain, $E_{\mathrm{c}} L \simeq 20 \mathrm{~V}$, donc $V(L) / E_{\mathrm{c}} L \ll 1$, et l'équation (73) montre, ainsi qu'il a déjà été signalé, que $S_{\mathrm{I}(\mathrm{G}-\mathrm{R})}$ est proportionnel à la puissance continue $I_{0} V(L)$ au point de polarisation, c'est-àdire croît linéairement avec la tension drain, en régime de saturation.

- Dans un FET submicronique, par exemple $L \simeq 0,5 \mu \mathrm{m}, E_{\mathrm{c}} L \simeq 0,5 \mathrm{~V}$, et $V(L) / E_{\mathrm{c}} L \gg 1$, et l'équation (73) montre que $S_{I(\mathrm{G}-\mathrm{R})}$ est proportionnel à $I_{0} / V(L)$, c'est-à-dire décroît lorsque la tension drain croît, en régime de saturation. Par conséquent, des composants courts ont un comportement totalement différent des composants longs.

Cependant, le comportement des composants courts n'est en fait pas tant caractérisé par des vạriations d'échelle ou de facteurs numériques, que par l'apparition de nouveaux phénomènes, qui n'ont aucun effet dans le cas de composants plus longs, et qui doivent être pris en compte dans des composants courts.

7.2 EFFETS DE DYNAMIQUE NON STATIONNAIRE. Les équations de transport, utilisées pour modéliser les composants longs, ne sont plus valables dans le cas des composants submicroniques. La raison en est que, lorsque la longueur devient aussi petite que quelques libres parcours moyens (le libre parcours moyen est la distance moyenne parcourue entre deux collisions), chaque porteur subit très peu de collisions en voyageant d'une électrode à l'autre, et par conséquent n'est pas en régime stationnaire. Il n'y a pas égalité entre la vitesse moyenne d'un porteur (moyenne temporelle), et la vitesse de dérive (moyenne à un instant donné, sur l'ensemble des porteurs). Il n'y a pas ergodicité, contrairement à ce qui se passe dans les composants longs, où chaque porteur subit un grand nombre de collisions.

Dans ces conditions, aux équations de transport classiques, on doit ajouter, non pas les relations $v=v(E)$ et $D=D(E)$, mais d'autres relations tenant compte d'un régime non stationnaire. Ces équations sont les équations dynamiques, qui sont des équations approchées décrivant, avec une bonne approximation, le transport non stationnaire, tel que survitesses ou sousvitesses, etc..., et qui donnent des résultats en accord raisonnable avec les solutions exactes de l'équation de Boltzmann, dans une grande variété de situations (températures, matériaux, conditions initiales, etc...) [19, 37, 38].

Ces équations sont de la forme :

$$
\left\{\begin{array}{l}
\mathrm{d}(m v) / \mathrm{d} t=(q E / \hbar)-(m v) / \tau_{\mathrm{p}}(\varepsilon) \\
\mathrm{d} \varepsilon / \mathrm{d} t=q E v-\left(\varepsilon-\varepsilon_{0}\right) / \tau_{\varepsilon}(\varepsilon)
\end{array}\right.
$$

où $v$ et $\varepsilon$ sont la vitesse de dérive et l'énergie moyenne, $\tau_{\mathrm{p}}(\varepsilon)$ et $\tau_{\varepsilon}(\varepsilon)$ sont les temps de relaxation de la quantité de mouvement et de l'énergie, supposés dépendre seulement de l'énergie moyenne à l'instant considéré. Ils peuvent être par conséquent déterminés en régime stationnaire à la même énergie, à partir des courbes $\varepsilon_{\mathrm{s}}\left(E_{\mathrm{s}}\right)$ et $v_{\mathrm{s}}\left(E_{\mathrm{s}}\right)$ l'indice $\mathrm{s}$ désigne le régime stationnaire), obtenues en résolvant l'équation de Boltzmann stationnaire dans le champ électrique uniforme $E_{\mathrm{s}}$. De façon plus précise, en égalant à 0 les dérivées par rapport au temps des équations (81), on obtient :

$$
\tau_{\mathrm{p}}(\varepsilon)=m v_{\mathrm{s}} /\left(q E_{\mathrm{s}}\right)
$$

et

$$
\tau_{\varepsilon}(\varepsilon)=\left(\varepsilon-\varepsilon_{0}\right) /\left(q E_{\mathrm{s}} v_{\mathrm{s}}\right)
$$

ce qui, transporté dans les équations (81), donne finalement, le long du champ électrique :

$$
\left\{\begin{array}{l}
\mathrm{d}(m v) / \mathrm{d} t=q\left[E-\left(v / \mu_{\mathrm{s}}\left(\varepsilon_{\mathrm{s}}=\varepsilon\right)\right)\right] \\
\mathrm{d} \varepsilon / \mathrm{d} t=q\left[E v-E_{\mathrm{s}}\left(\varepsilon_{\mathrm{s}}=\varepsilon\right) v_{\mathrm{s}}\left(\varepsilon_{\mathrm{s}}=\varepsilon\right)\right]
\end{array}\right.
$$

où $\mu_{\mathrm{s}}\left(\varepsilon_{\mathrm{s}}\right)=v_{\mathrm{s}}\left(\varepsilon_{\mathrm{s}}\right) / E_{\mathrm{s}}\left(\varepsilon_{\mathrm{s}}\right)$. A un instant donné $t$, on suppose connus la vitesse de dérive $v=v(t)$, et l'énergie moyenne $\varepsilon=\varepsilon(t)$. Les courbes stationnaires donnent le champ $E_{\mathrm{s}}$ et la vitesse $v_{\mathrm{s}}$ correspondant à l'énergie $\varepsilon_{\mathrm{s}}=\varepsilon(t)$. Ces valeurs, portées dans les équations (82), donnent $\mathrm{d}(m v) / \mathrm{d} t$ et $\mathrm{d} \varepsilon / \mathrm{d} t$, et par conséquent $v(t+\Delta t)$ et $\varepsilon(t+\Delta t)$. Une légère modification intervient lorsqu'on étudie l'état stationnaire le long d'un composant : dans les équations ci-dessus, un déplacement $\Delta x$ est associé à un incrément de temps $\Delta t=\Delta x / v$.

En ce qui concerne le bruit, on considère aussi qu'il dépend, non pas du champ électrique local, mais de l'énergie locale. Ainsi, par exemple, la source de bruit de diffusion est donnée par [21, 22] :

$$
K(x)=4 q^{2} n(x) D(\varepsilon(x)) .
$$

Ces effets non stationnaires ont été pris en compte pour modéliser des dispositifs courts, et en particulier divers types de FETs [20-27]. Par exemple, Carnez et al. [21] on montré que le fait de tenir compte des effets non stationnaires, pouvait donner lieu à une importante diminution du facteur de bruit de MES-FETs GaAs à grille courte, ainsi qu'il ressort de la figure 10 tirée de la référence [21].

Toutefois, une modélisation «classique » en 


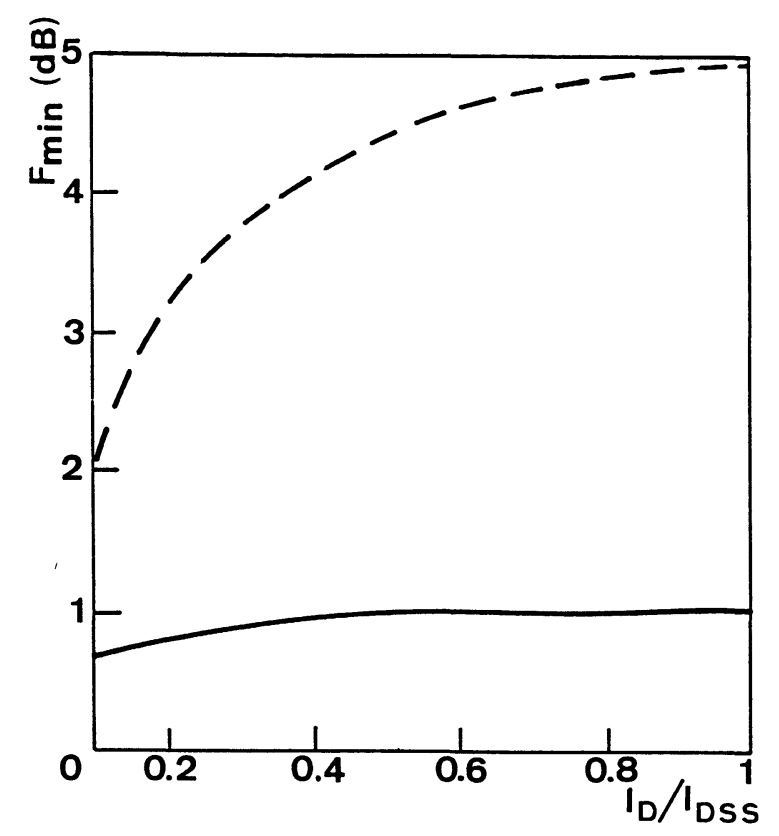

Fig. 10. - Variation, en fonction du courant drain, du facteur de bruit minimal $F_{\text {min }}$ d'un MES-FET GaAs, de longueur de grille $0,4 \mu \mathrm{m}$, en tenant compte (trait plein) et sans tenir compte (traits discontinus) des effets de dynamique non stationnaire [21].

[Variation, versus the drain current, of the minimum noise figure $F_{\min }$ of a GaAs MES-FET, with $0.4 \mu \mathrm{m}$ gate length, when taking (full line), and not taking (dashed line) into account, non stationary effects [21].]

régime stationnaire de porteurs chauds, effectuée [39] sur un TEG-FET GaAs/GaAlAs, en utilisant la méthode décrite dans l'exemple du $\$ 5.3$ ci-dessus, sans tenir compte d'effets non stationnaires, a donné des résultats en bon accord avec l'expérience. De la même manière, un bon accord a été obtenu sur une diode $n^{+}-n-n^{+}$GaAs, en utilisant la méthode décrite au $\$ 5.2$ sans tenir compte non plus d'effets non stationnaires [40].

Cependant, très peu d'expériences ont été effectuées sur des composants submicroniques [38-42], et il est en tout état de cause toujours très difficile de connaître de manière suffisamment précise les données technologiques et physiques d'un tel composant [52]. Bien évidemment, dans les composants courts aussi apparaissent des effets parasites, tel du bruit en $1 / f[43]$ aux interfaces.

6.3 Corrélations SPATIAles DES SOURCES DE BRUIT. - On a supposé, dans tout ce qui précède, que les sources de bruit en deux points voisins étaient décorrélées. Il a été récemment démontré $[44,45]$ que ceci n'était pas vrai, sur des distances de l'ordre de quelques libres parcours moyens. En effet, la source de bruit est la transformée de Fourier de la fonction de corrélation de la densité de courant (cf. Eqs. (3) et (9)), c'est-à-dire en particulier des vitesses des porteurs. Sur des distances plus courtes que la distance entre deux collisions, la vitesse d'un porteur est inchangée ( $\mathrm{si} F=0$ ), donc les vitesses en ces points sont strictement corrélées.

Moyennant les hypothèses suivantes :

a) Deux porteurs différents sont décorrélés.

b) Les mécanismes de collision sont isotropes (interactions avec les phonons acoustiques, optiques non polaires, intervallées), on peut écrire que la probabilité pour qu'il y ait corrélation, entre la vitesse $\mathbf{v}_{\mathrm{i}}(\mathbf{r}, t)$ du porteur numéro i situé au point $\mathbf{r}$ à l'instant $t$, et sa vitesse $\mathbf{v}_{\mathrm{i}}\left(\mathbf{r}^{\prime}, t+\theta\right)$ au point $\mathbf{r}^{\prime}$ à l'instant $t+\theta$, vaut :

-1 avec la probabilité $p_{1} p_{2}$

-0 avec la probabilité $\left(1-p_{1} p_{2}\right)$

avec :

$-p_{1}=$ probabilité que le porteur numéro $i$ n'ait subi aucune collision dans l'intervalle de temps $\theta$. $-p_{2}=$ probabilité pour que, dans l'intervalle de temps $\theta$, le porteur se soit déplacé de $\mathbf{r}$ en $\mathbf{r}^{\prime}$ à $\mathrm{d}^{3} r^{\prime}$ près.

Soit k l'état du porteur à l'instant $t$. En présence d'un champ électrique $\mathbf{E}$, son état à l'instant $t+u$ est, s'il n'y a pas eu collision entre $t$ et $t+u$ :

$$
\mathbf{k}(u)=\mathbf{k}+(q \mathbf{E} u / \hbar)
$$

Si $P\left(\mathbf{k}, \mathbf{k}^{\prime}\right)$ est la probabilité de transition par unité de temps entre les états $\mathbf{k}$ et $\mathbf{k}^{\prime}$, la probabilité pour que, pendant l'intervalle de temps $\mathrm{d} u$, le porteur dans l'état $\mathbf{k}(u)$ subisse une collision, s'écrit :

$$
\mathrm{d} u / \tau(\mathbf{k}(u))=\int P\left(\mathbf{k}(u), \mathbf{k}^{\prime}\right) \mathrm{d}^{3} k^{\prime} .
$$

On a alors :

$$
\left\{\begin{array}{l}
p_{1}=\exp -\int_{0}^{\theta} \mathrm{d} u / \tau(\mathbf{k}(u)) \\
p_{2}=\mathrm{d}^{3} r^{\prime} \cdot \delta\left(\mathbf{r}^{\prime}-\mathbf{r}-\int_{0}^{\theta} \mathrm{d} u \mathbf{v}(\mathbf{k}(u))\right) .
\end{array}\right.
$$

On obtient alors, en présence d'un champ électrique $E$, pour fonction de corrélation de la densité de courant entre les directions $\alpha$ et $\beta$ :

$$
\begin{aligned}
C_{j \alpha \beta}\left(\mathbf{r}, \mathbf{r}^{\prime}, \theta\right)=q^{2} n\left[\int \Delta v_{\alpha} \Delta v_{\beta} f(\mathbf{k}, E)(\exp \right. & \left.-\int_{0}^{\theta} \mathrm{d} u / \tau(\mathbf{k}(u))\right) \times \\
& \left.\times \delta\left(\mathbf{r}^{\prime}-\mathbf{r}-\int_{0}^{\theta} \mathrm{d} u \mathbf{v}(\mathbf{k}(u))\right)\right] / \int f(\mathbf{k}, E) \mathrm{d}^{3} k
\end{aligned}
$$


avec

$$
\begin{aligned}
v_{\alpha} & =v_{\alpha}(\mathbf{k})-v_{0 \alpha}(\mathbf{k}) \\
\Delta v_{\beta} & =v_{\beta}(\mathbf{k}(\theta))-v_{0 \beta}(\mathbf{k}, \theta) . \\
\mathbf{k}(u) & =\mathbf{k}+(q \mathrm{E} u / \hbar)
\end{aligned}
$$

Dans ces équations, $f(\mathbf{k}, E)$ est la fonction de distribution stationnaire en régime de porteurs chauds, $\mathbf{v}(\mathbf{k})=(1 / \hbar) \nabla_{k} \varepsilon(\mathbf{k}), \mathbf{v}_{0}(\mathbf{k})$ est la vitesse de dérive, c'est-à-dire la moyenne de $\mathbf{v}(\mathbf{k})$ sur la fonction $f(\mathbf{k}, E)$, et $\mathbf{v}_{0}(\mathbf{k}, \theta)$ est la moyenne, à l'instant $\theta$, des vitesses des porteurs qui se trouvaient dans l'état $\mathbf{k}$ à l'instant $t(u=0)$.

En régime ohmique [44] $(E=0)$, les porteurs ne sont pas accélérés pendant un vol libre, donc $\mathbf{k}(u)=\mathbf{k}$ (cf. Eqs. 86)) et l'équation (85) s'écrit :

$$
\begin{aligned}
C_{j \alpha \beta}\left(\mathbf{r}, \mathbf{r}^{\prime}, \theta\right) & = \\
= & {\left[\int v_{\alpha} v_{\beta} f_{0}(\mathbf{k})[\exp (-\theta / \tau(k))] \times\right.} \\
\times & \left.\delta\left(\mathbf{r}^{\prime}-\mathbf{r}-\theta v\right) \mathrm{d}^{3} k\right] / \int f_{0}(\mathbf{k}) \mathrm{d}^{3} k
\end{aligned}
$$

où $f_{0}(\mathbf{k})$ est la fonction de distribution à l'équilibre thermodynamique. Le terme de source de bruit s'obtient en prenant la transformée de Fourier de l'équation (87) :

$$
\begin{aligned}
& S_{j \alpha \beta}\left(\mathbf{r}, \mathbf{r}^{\prime}, f\right)= \\
& \quad=4 \int_{0}^{\infty} C_{j \alpha \beta}\left(\mathbf{r}, \mathbf{r}^{\prime}, \theta\right) \cos (2 \pi f \theta) \mathrm{d} \theta
\end{aligned}
$$

Lorsque $\alpha=\beta=x, \mathbf{r}-\mathbf{r}^{\prime}=\mathbf{R}=\{R, 0,0\}$, les équations (87) et (88) donnent $S_{j \|}(R, f)$, qui peut être calculée numériquement pour un semiconducteur donné. La figure 11 montre $S_{j \|}(R, f)$ dans Si-p à l'équilibre thermodynamique, en fonction de la distance $R$, à différentes fréquences [44]. Le bruit de diffusion est blanc en dessous de $10^{11} \mathrm{~Hz}$. Il est clair, sur la figure 11 , que $S_{j}$ est nul en $R=0$, ce qui est tout à fait contraire à l'hypothèse habituelle (cf. Eq. (10)). Ceci est dû à ce que la corrélation ellemême est nulle en $\mathbf{r}-\mathbf{r}^{\prime}=\mathbf{R}=\mathbf{0}$. En effet, considérons un porteur en $\mathbf{r}$ à l'instant $t$, sa vitesse vaut $\mathbf{v}_{i}(\mathbf{r}, t) ;$ si $\mathbf{v}_{i}(\mathbf{r}, t)$ est non nul, à l'instant $t+\theta$ le porteur n'est plus en $\mathbf{r}$, seul peut se trouver en $\mathbf{r}$ à l'instant $t+\theta$ un porteur $j \neq i$, dont la vitesse $\mathbf{v}_{j}$ est par hypothèse décorrélée de $\mathbf{v}_{i} ;$ si $\mathbf{v}_{i}(\mathbf{r}, t)=0$, alors $\mathbf{v}_{i}(\mathbf{r}, t+\theta)=0$ (s'il n'y a pas eu collision entre temps), et là encore $\mathbf{v}_{i}(\mathbf{r}, t) \cdot \mathbf{v}_{i}(\mathbf{r}, t+\theta)=0$, donc la fonction de corrélation $C_{j}(R=0, \theta)$ vaut 0 , ainsi par conséquent que sa transformée de Fourier $S_{j}(R=0, f)$. La figure 11 nous montre enfin que $S_{j}(R, f)$ est maximale pour $R \simeq 100 \AA$, c'est-à-dire pour une distance de l'ordre du libre parcours moyen, et que $S_{j}(R, f)$ s'étend sur quelques libres parcours moyens.

Soit $\tau$ la durée du libre parcours moyen, et $\mathbf{k}_{\text {th }}$ l'état tel que $v_{\text {th }}=\left|\mathbf{v}\left(\mathbf{k}_{\text {th }}\right)\right|$, où $v_{\text {th }}$ est la vitesse

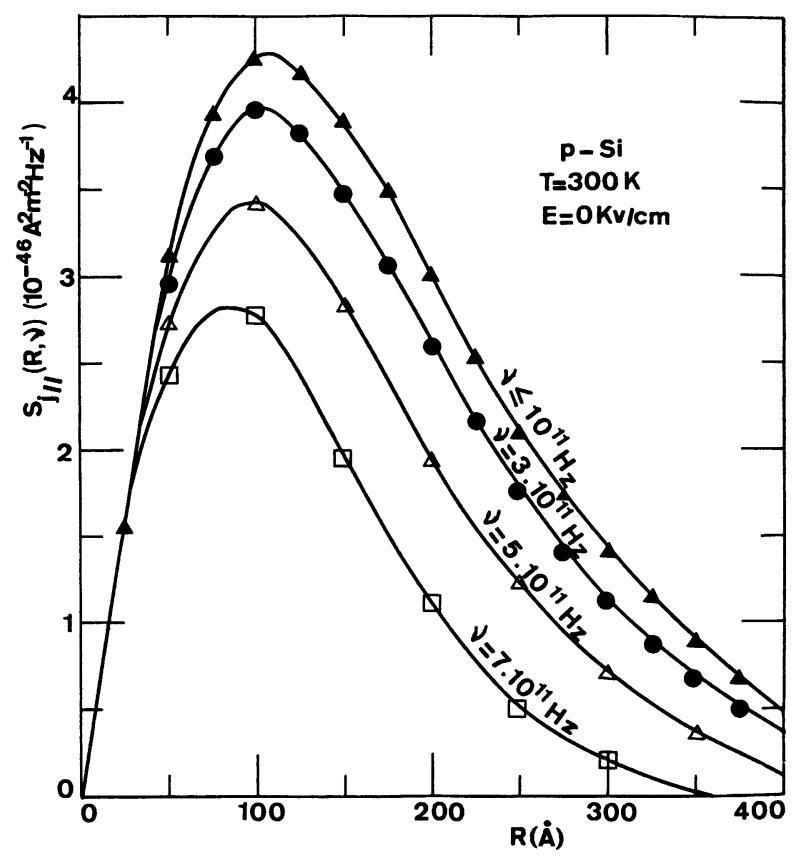

Fig. 11. - Variation, en fonction de la distance $R$, du terme de source de bruit longitudinal $S_{j l}(R, f)$, à diverses fréquences $f$, dans $\mathrm{Si}-\mathrm{p}, T=300 \mathrm{~K}, E=0$, mettant en évidence les corrélations spatiales des sources de bruit de diffusion [44].

[Variation, versus the distance $R$, of the longitudinal noise source term $S_{j}(R, f)$, at various frequencies $f$, in p-type $\mathrm{Si}, T=300 \mathrm{~K}, E=0$, exhibiting space correlations of the diffusion noise sources [44].]

d'agitation thermique. Pour $|\mathbf{k}| \gg\left|\mathbf{k}_{\text {th }}\right|$, on a $f_{0}(\mathbf{k}) \simeq 0$, et pour $\theta \gg \tau, \exp -\theta / \tau(\mathbf{k}) \simeq 0$. Par conséquent, dans les composants longs, tels que $\left|\mathbf{r}-\mathbf{r}^{\prime}\right| \gg v_{\text {th }}$, on peut écrire :

$$
\delta\left(\mathbf{r}^{\prime}-\mathbf{r}-\mathbf{v} \boldsymbol{\theta}\right) \simeq \delta\left(\mathbf{r}^{\prime}-\mathbf{r}\right)
$$

de sorte que les équations (87) et (88) deviennent :

$$
\begin{aligned}
S_{j \alpha \beta}\left(\mathbf{r}, \mathbf{r}^{\prime}, f\right) & \simeq K_{\alpha \beta}(\mathbf{r}, f) \delta\left(\mathbf{r}^{\prime}-\mathbf{r}\right) \\
K_{\alpha \beta}(\mathbf{r}, f) & =4 q^{2} n D_{\alpha \beta}(f) \\
D_{\alpha \beta}(f) & =\left\langle\tau(\mathbf{k}) v_{\alpha} v_{\beta} /\left[1+\omega^{2} \tau^{2}(\mathbf{k})\right]\right\rangle
\end{aligned}
$$

où les crochets représentent la moyenne sur la fonction de distribution. Ceci est l'expression habituelle du coefficient de diffusion, pour des interactions isotropes.

On remarquera que, en présence d'un champ électrique $E \neq 0$, d'après l'équation (85), $S_{j}$ est un tenseur diagonal, avec en général $S_{j \|} \neq S_{j \perp}$. A champ nul au contraire, $f_{0}(\mathbf{k})$ possède une symétrie de révolution, et l'équation (87) montre que $S_{j}$ est un scalaire.

Nous avons vu dans ce paragraphe, que les sources de bruit de diffusion étaient corrélées sur quelques libres parcours moyens : ceci devrait être pris en compte dans les composants submicroniques. 
De plus, la corrélation entre deux points dépend de leur distance, et donc la corrélation entre deux tranches dépend des couples de points considérés. En conséquence, il semble que le modèle unidimensionnel soit en défaut.

Ce qui vient d'être dit concerne les semiconducteurs non polaires. Dans les composés III-V et II-VI, les collisions prédominantes se font sur les phonons optiques polaires : ce processus, comme les interactions sur les impuretés, n'est pas isotrope. Par conséquent, l'état $\mathbf{k}^{\prime}$ après collision est dirigé préférentiellement dans la direction de l'état $\mathbf{k}$ incident. Il s'ensuit qu'une collision ne décorrèle pas totalement les vitesses. On peut donc s'attendre à ce que la distance, sur laquelle une corrélation existe, soit encore plus grande que dans les semiconducteurs non polaires.

6.4 Changement DE NATURE DU BRUIT. - Considérons un composant avec du bruit de diffusion. En fait, le bruit de grenaille aussi est présent, superposé au bruit de diffusion. Dans un composant long (longueur bien plus grande que quelques libres parcours moyens), chaque porteur subit de nombreux chocs en voyageant d'une électrode à l'autre, et le bruit de diffusion est largement prédominant : $S_{\text {I(diff) }} \gg S_{\text {I(grenaille) }}$. Au contraire, dans les composants de longueur approchant le libre parcours moyen, chaque porteur voyage d'une électrode à l'autre presque sans subir de collision, donc le bruit de diffusion disparaît, et seul le bruit de grenaille subsiste. Cette diminution du bruit de diffusion, due aux courtes distances responsables des effets non stationnaires, est mise en évidence sur la figure 10, par la diminution du facteur de bruit du FET, par rapport à la valeur obtenue en utilisant les équations « classiques » de transport stationnaire. Des tentatives ont été faites, ces dernières années [46], pour mettre en évidence de tels effets dans des dipôles. Elles ont toutes échoué car, dans ces composants, les effets parasites, tels la résistance de contact, le bruit en $1 / f$, etc..., masquent une possible variation due à un transport ballistique dans la région active. Des FETs, ou d'autres composants, où une électrode joue le rôle de sonde, seraient probablement mieux adaptés à mettre en évidence de tels effets, ainsi qu'il a été suggéré quelques années plus tôt [35].

6.5 EFFETS DE DISCRÉTISATION. - Lorsque les dimensions deviennent très petites, le schéma de bandes classique n'est plus valable. Il apparaît des niveaux d'énergie discrets. Ceci se produit, par exemple, dans les couches épitaxiées, les superréseaux, les couches d'inversion, etc... Un certain nombre de conséquences en découlent.

Si les niveaux d'énergie doivent être considérés individuellement, et non statistiquement, le comportement dépend de la structure fine des niveaux. Ceci a été mis en évidence [47] en étudiant un modèle unidimensionnel de membrane poreuse. Les ions sont supposés sauter d'un puits de potentiel à un puits adjacent, et le spectre de bruit dépend alors fortement de la distribution et des valeurs des énergies des puits.

Dans les couches minces où les niveaux d'énergie sont discrets, les probabilités de transition par unité de temps diffèrent des matériaux en volume, et les coefficients de transport sont modifiés en conséquence [19]. Une récente simulation de Monte Carlo, faite sur des puits quantiques $\mathrm{GaAs} / \mathrm{GaAlAs}$, a montré que le coefficient de diffusion $D(E)$ dépendait fortement de l'épaisseur des couches [48].

Dans les structures petites, la densité de porteurs doit être élevée, et par conséquent il faut tenir compte des interactions porteur-porteur, ainsi que de la dégénérescence. De plus, le modèle corpusculaire des porteurs doit être remis en question, puisque leur longueur d'onde $\lambda=2 \pi / k$, avec $k \simeq 10^{9} \mathrm{~m}^{-1}$, est de l'ordre de $60 \AA$, ce qui n'est plus du tout négligeable devant l'épaisseur des couches.

Finalement, la caractéristique électrique d'un composant, comme on l'a vu en particulier en ce qui concerne le bruit, est une certaine moyenne de valeurs de paramètres de transport sur tout le dispositif. Par conséquent, un petit défaut noyé dans un composant de grandes dimensions apporte une perturbation localisée, qui, une fois moyennée sur l'ensemble du composant, peut avoir un effet global négligeable. Par contre, le même défaut, dans un composant submicronique, occupe la plus grande partie du volume du composant, et par conséquent la perturbation qu'il produit affecte le comportement du composant tout entier. Les composants submicroniques sont bien plus sensibles aux défauts que les composants longs : ceci ne peut pas être prédit ou modélisé facilement.

\section{Conclusion.}

Ces dernières années, de puissantes méthodes ont été développées, pour modéliser le bruit de composants, orientées principalement autour de la méthode du champ d'impédances. Grâce à une approche numérique, il est maintenant possible de modéliser le bruit d'une grande variété de composants unidimensionnels en régime de conduction unipolaire. Ces méthodes peuvent, en principe, s'appliquer aussi aux mécanismes de conduction ambipolaire, bien que ceci n'ait pas été fait jusqu'à présent, à ma connaissance.

L'extension de ces méthodes, à des structures biou tridimensionnelles, soulève des problèmes, de nature au moins technologique (temps de calcul, occupation de place mémoire, etc...), et probablement même de nature fondamentale.

Les méthodes décrites ici peuvent être (et ont été) appliquées à des composants submicroniques, en 
incluant les effets non stationnaires telles la survitesse. Cependant, dans de telles structures, un grand nombre de problèmes ne sont encore pas résolus, ainsi que je l'ai souligné notamment aux $\$ \S 6.3$ et 6.5 ci-dessus. La plupart de ces problèmes fondamentaux (principalement ceux liés aux effets de discrétisation), joints aux incertitudes considérables sur les valeurs des paramètres technologiques et électriques des couches (concentration de dopages, défauts, inhomogénéités, etc...), ne permettent même pas, bien souvent, de modéliser les caractéristiques du "premier ordre", telles que les profils de champs électriques, de concentration de porteurs, les carac- téristiques $I-V$ et petits signaux, les fréquences de coupure, etc... Or, il importe de garder à l'esprit qu'il est totalement illusoire de prétendre modéliser le bruit tant qu'on n'est pas capable de modéliser avec une bonne précision les caractéristiques du premier ordre. On conçoit alors que, dans ces conditions, la modélisation du bruit de composants submicroniques soit un champ d'investigations très ouvert.

Inversement, l'étude expérimentale du bruit de composants submicroniques est possible, et peut apporter des informations aussi bien sur des aspects technologiques que sur des paramètres physiques responsables du bruit observé.

\section{Bibliographie}

[1] VAN DER ZIEL, A., Noise : Sources, Characterization, Measurement, Prentice Hall, Englewood Cliffs, NJ 1970.

[2] Van Vliet, K. M., Friedmann, A., Zijlstra, R. J. J., Gisolf, A., VAN Der Ziel, A., J. Appl. Phys. 46 (1975) 1804 ; J. Appl. Phys. 46 (1975) 1814.

[3] Nougier, J. P., Proc. 6th Int. Conf. on Noise in Physical Systems, Washington 1981, ed. by P. H. E. Meijer, R. D. Mountain, R. J. Soulen, National Bureau of Standards, Special Publication No. 614, p. 397 (1981).

[4] Van Der Ziet, A., Van Vliet, K. M., Solid State Electron. 11 (1968) 508.

[5] Van Vliet, K. M., Solid State Electron. 13 (1970) 649.

[6] Nougier, J. P., in Noise in Physical Systems, Springer Ser. Electrophys., Vol. 2, ed. D. Wolf (Springer) 1978, p. 71.

[7] Nougier, J. P., in Physics of Non Linear Transport in Semiconductors, ed. D. K. Ferry, J. R. Barker, C. Jacoboni (Plenum Press, New York) 1980, p. 415.

[8] Nougier, J. P., Vaissière, J. C., Gasquet, D., Moatadid, A., J. Appl. Phys. 52 (1981) 5683.

[9] Nougier, J. P., Appl. Phys. Lett. 33 (1978) 89.

[10] Van Vliet, K. M., Friedmann, A., ZiJlstra, R. J. J., Gisolf, A., VAN Der Ziel, A., J. Appl. Phys. 46 (1975) 1814.

[11] VAN Der Ziel, A., Jindal, R., Kim, S. K., PARK, H., NougIER, J. P., Solid State Electron. 22 (1979) 177.

[12] Hooge, F. N., Phys. Lett. 29A (1969) 139.

[13] Vandamme, L. K. J., in Noise in Physical Systems and $1 / f$ Noise, ed. M. Savelli, G. Lecoy, J. P. Nougier (North-Holland, Amsterdam) 1983, p. 183.

[14] Gasquet, D., Fadel, M., Nougier, J. P., in Noise in Physical Systems and $1 / f$ Noise, ed. M. Savelli, G. Lecoy, J. P. Nougier (North-Holland, Amsterdam) 1983, p. 15.

[15] Shockley, W., Copeland, J. A., James, R. R., in Quantum Theory of Atoms, Molecules and the Solid State, ed. P. Lowdin (Academic Press, New York) 1966, Chap. 8.
[16] Rigaud, A., Nicolet, M. A., Savelli, M., Phys. Status Solidi A 18 (1973) 531.

[17] Gasquet, D., Tijani, H., Nougier, J. P., in Noise in Physical Systems and $1 / f$ Noise, ed. $M$. Savelli, G. Lecoy, J. P. Nougier (North-Holland, Amsterdam) 1983, p. 165.

[18] Vaissière, J. C., Thèse de Doctorat ès Sciences, Montpellier, 1986.

[19] Reggiani, L. in Hot Electron Transport in Semiconductors, ed. L. Reggiani, Topics in Appl. Phys., Vol. 58 (Springer Berlin Heidelberg) 1985.

[20] Carnez, B., Cappy, A., Salmer, G., Constant, E., Acta Electronica 23 (1980) 165.

[21] Carnez, B., Cappy, A., Fauquembergue, R., Constant, E., SAlmer, G., IEEE Trans. ED28 (1981) 784.

[22] Cappy, A., Fauquembergue, R., Constant, E., Salmer, G., Carnez, B., Inst. Phys. Conf. Ser. 56 Chap. 3 (1981) 175.

[23] Allamando, E., Salmer, G., Constant, E., Radhy, N. E., CAPPy, A., Carnez, B., in Noise in Physical Systems and $1 / f$ Noise, ed. M. Savelli, G. Lecoy, J. P. Nougier (North-Holland, Amsterdam) 1983, p. 177.

[24] Allamando, E., Radhy, N. E., Salmer, G., Physica B 129 (1985) 390.

[25] Cappy, A., Schortgen, M., Salmer, G., IEEE Trans. EDL-6 (1985) 270.

[26] Cappy, A., Vanoverschelde, A., Schortgen, M., Versnaeyeen, C., Salmer, G., IEEE Trans. ED-32 (1985) 2787.

[27] Cappy, A., Vanoverschelde, A., Schortgen, M., Versnaeyeen, C., Salmer, G., Physica B 129 (1985) 380.

[28] Brewitt-Taylor, C. R., Robson, P. N., Sitch, J. E., Proc. 8th European Microwave Conference (Paris 1978), p. 415:

[29] Sodini, D., Rigaud, D., Proc. 6th Int. Conf. on Noise in Physical Systems, Washington 1981, ed. P. H. E. Meijer, R. D. Mountain, R. J. Soulen, National Bureau of Standards, Special Publication No. 614 (1981).

[30] Gisolf, A., ZiJlstra, R. J. J., Solid State Electron. 17 (1974) 839. 
[31] Bosmann, G., Zijlstra, R. J. J., Phys. Lett. A 71 (1979) 464.

[32] Nougier, J. P., Gasquet, D., Vaissière, J. C., Bilger, H. R., in Noise in Physical Systems, Springer Ser. Electrophys., Vol. 2, ed. D. Wolf (Springer Berlin Heidelberg) 1978 p. 110.

[33] Nougier, J. P., Moatadid, A., Vaissière, J. C., Gasquet, D., Physica B 134 (1985) 260.

[34] Nougier, J. P., Vaissière, J. C., Gasquet, D., in Proc. 6th Int. Conf. on Noise in Physical Systems, Washington 1981, ed. P. H. E. Meijer, R. D. Mountain, R. J. Soulen, National Bureau of Standards, Special Publication No. 614 (1981), p. 42.

[35] Hesto, P., Vaissière, J. C., Castagne, R., NouGIER, J. P., J. Physique Colloq. 42 (1981) 235.

[36] Nougier, J. P., Moatadid, A., Vaissière, J. C., Gasquet, D., in Noise in Physical Systems and $1 / f$ Noise 1985, ed. A. D'Amico, P. Mazzetti (North-Holland Amsterdam) 1986, p. 105.

[37] Nougier, J. P., Vaissière, J. C., Gasquet, D., Zimmermann, J., Constant, E., J. Appl. Phys. 52 (1981) 825.

[38] Reggiani, L., Vaissière, J. C., Nougier, J. P., Gasquet, D., J. Physique Colloq. 42 (1981) 357.

[39] Whiteside, C. F., Bosman, G., Van Der Ziel, A., KOPP, W., MORKOC, H., in Noise in Physical Systems and $1 / f$ Noise, ed. A. D'Amico, P. Mazzetti (North-Holland, Amsterdam) 1986, p. 167.
[40] Andrian, J., Bosman, G., VAN Der Ziel, A., VAN Vliet, C. M., in Noise in Physical Systems and $1 / f$ Noise, ed. A. D'Amico, P. Mazzetti (North-Holland, Amsterdam) 1986, p. 171.

[41] Delagebeaudeuf, D., Linh, N. T., IEEE Trans. ED-29 (1982) 955.

[42] Mishra, O. K., Palmateer, S. C., Chao, P. C., Smith, P. M., Hwang, J. C. M., IEEE Trans. EDL-6 (1985) 142.

[43] Duh, K. H., Van Der Ziel, A., Morkoc, H., IEEE EDL-4 (1983) 10.

[44] Nougier, J. P., Gontrand, C., Vaissière, J. C., in Noise in Physical Systems and $1 / f$ Noise, ed. M. Savelli, G. Lecoy, J. P. Nougier (North-Holland, Amsterdam) 1983, p. 15.

[45] Nougier, J. P., Vaissière, J. C., Gontrand, C., Phys. Rev. Lett. 51 (1983) 513.

[46] Noise in Physical Systems and $1 / f$ Noise, ed. M. Savelli, G. Lecoy, J. P. Nougier (NorthHolland, Amsterdam) 1983.

[47] Frehland, E., Biophys. Struct. Mech. 7 (1980) 1.

[48] Van Rheenen, A. D., Bosman, G., in Noise in Physical Systems and $1 / f$ Noise, ed. A. D'Amico, P. Mazzetti (North-Holland, Amsterdam) 1986, p. 163.

[49] Rolland, M., Nougier, J. P., Gasquet, D., AlaBEDRA, R., Solid State Electron. 20 (1977) 323.

[50] CAPPY, A., Thèse de Doctorat ès Sciences, Lille, 1986:

[51] Hill, G., Robson, P. N., Fawcett, W., J. Appl. Phys. 50 (1979) 356. 